\title{
Rational Remodeling of Atypical Scaffolds for the Design of Photoswitchable Cannabinoid Receptor Tools
}

\author{
Tao Hu$u^{[a][b][c]+}$, Guoxun Zheng ${ }^{[a]+}$, Dongxiang Xue ${ }^{[a]}$, Simeng Zhao[a], Fei Li ${ }^{[a]}$, Fang Zhou ${ }^{[a]}$, Fei Zhao ${ }^{[a]}$, \\ Linshan Xie ${ }^{[a][b]}$, Cuiping Tian ${ }^{[a]}$, Tian Hua ${ }^{[a] b]}$, Suwen Zhao ${ }^{[a][b]}$, Yueming Xud ${ }^{[a]}$, Guisheng Zhong ${ }^{[a][b]}$, \\ Zhi-Jie Liu ${ }^{[a][b]}$, Alexandros Makriyannis ${ }^{[d]}$, Raymond C. Stevens ${ }^{[a] b][e]}$, and Houchao Tao*[a] \\ [a] iHuman Institute, ShanghaiTech University, Pudong, Shanghai 201210, China \\ ${ }^{[b]}$ School of Life Science and Technology, ShanghaiTech University, Pudong, Shanghai 201210, China \\ ${ }^{[c]}$ CAS Center for Excellence in Molecular Cell Science, Shanghai Institute of Biochemistry and Cell Biology, Chinese \\ Academy of Sciences, Shanghai, China; University of Chinese Academy of Sciences, Beijing 100049, China \\ [d] Center for Drug Discovery, Department of Pharmaceutical Sciences and Department of Chemistry and Chemical Biology, \\ Northeastern University, Boston, MA 02115, USA \\ ${ }^{[\mathrm{e}]}$ Departments of Biological Sciences and Chemistry, Bridge Institute, USC Michelson Center for Convergent Bioscience, \\ University of Southern California, CA 90089, USA. \\ ${ }^{[+]}$These authors contributed equally.
}

*Corresponding author

Houchao Tao, taohch@ shanghaitech.edu.cn. 


\section{Table of Contents}

\begin{tabular}{|c|c|c|}
\hline Table S1 & Overview of published transformation of azosters & S3 \\
\hline Table S2 & Ligand statistics from different database & S8 \\
\hline Figure S1 & Residue distribution within the range of $4 \AA$ of typical azologization fragments & S9 \\
\hline Figure S2 & Residue distribution within the range of $4 \AA$ of atypical azologization fragments & S10 \\
\hline Figure S3 & Molecular dynamic simulation of CB2 in complex with AM10257 & S11 \\
\hline Figure S4 & Ligand statistics for $\mathrm{CB} 2$ & S12 \\
\hline Figure S5 & Representative CB2 ligands with adamantyl group & $\mathbf{S 1 3}$ \\
\hline Figure S6 & UV-vis spectra for photoswitchable ligands & S14 \\
\hline Table S3 & The maximum absorption wavelength for trans and cis isomers in HBSS & S14 \\
\hline Figure S7 & Thermal isomerization of photoswitchable ligands & S15 \\
\hline Table S4 & Half-life of $c i s$-form AzoLigs at room temperature & S15 \\
\hline Table S5 & Ratio of cis-AzoLig 9 under different illumination time determined by ${ }^{1} \mathrm{H}$ NMR & S16 \\
\hline Figure S8 & Full HPLC chromatograms of AzoLig 9 under different illumination time & S17 \\
\hline Table S6 & Ratio of $c i s$-AzoLig 9 under different illumination time determined by HPLC & S17 \\
\hline Figure S9 & Chemical structures of AM10257 and SR144528 & S18 \\
\hline Table S7 & Pharmacological activity of AM10257 and SR144528 for CB2 & S18 \\
\hline Figure S10 & Molecular dynamic simulation of CB2 with AzoLig 9 & S19 \\
\hline Figure S11 & Binding mode of $c i s$-form of ortho-substituted AzoLigs in CB2 & $\mathbf{S 2 0}$ \\
\hline Figure S12 & Binding mode of cis-AzoLigs with different azo position in CB2 & $\mathbf{S 2 1}$ \\
\hline Figure $\mathbf{S 1 3}$ & Binding mode of AzoLig 9 in CB1 & $\mathbf{S 2 2}$ \\
\hline \multicolumn{2}{|c|}{ NMR Spectra of Final Photoswitchable Ligands } & $\mathbf{S 2 3}$ \\
\hline \multicolumn{2}{|c|}{ Purification Characterization } & S36 \\
\hline \multicolumn{2}{|l|}{ References } & S42 \\
\hline
\end{tabular}




\section{Supplemental Figures and Tables}

Table S1. Overview of published transformation of azosters. ${ }^{a}$

\begin{tabular}{|c|c|c|c|c|}
\hline No. & Targets/Function & Parent ligands & azoligands & Classes \\
\hline 1 & GABAA & Gz-i1 & & $1^{1}$ \\
\hline 2 & Microtubules & $\begin{array}{l}\text { Combretastatin A-4 } \\
(\mathrm{CA} 4)\end{array}$ & & $1^{2}$ \\
\hline 3 & GLP-1R & & & $1^{3}$ \\
\hline 4 & $\begin{array}{l}\text { Human serotonin } \\
\text { transporter (hSERT) }\end{array}$ & & & $1^{4}$ \\
\hline 5 & $\begin{array}{l}\text { Excitatory amino acid } \\
\text { transporters } \\
\text { GltTk }\end{array}$ & & & $1^{5,6}$ \\
\hline 6 & $\begin{array}{l}\text { Nicotinic acetylcholine } \\
\text { receptors (nAChRs) }\end{array}$ & & & $1^{7}$ \\
\hline 7 & F1Fo-ATP synthase & & & $1^{8}$ \\
\hline 8 & $\begin{array}{l}\text { REarranged during } \\
\text { Transfection (RET) }\end{array}$ & & & $1^{9}$ \\
\hline 9 & GPR40 & & & $1^{10}$ \\
\hline 10 & mGlu5 & & & $1^{11,12}$ \\
\hline 11 & Sirtuin 2 & & & $1^{13}$ \\
\hline 12 & Sirtuin 2 & & & $1^{13}$ \\
\hline
\end{tabular}




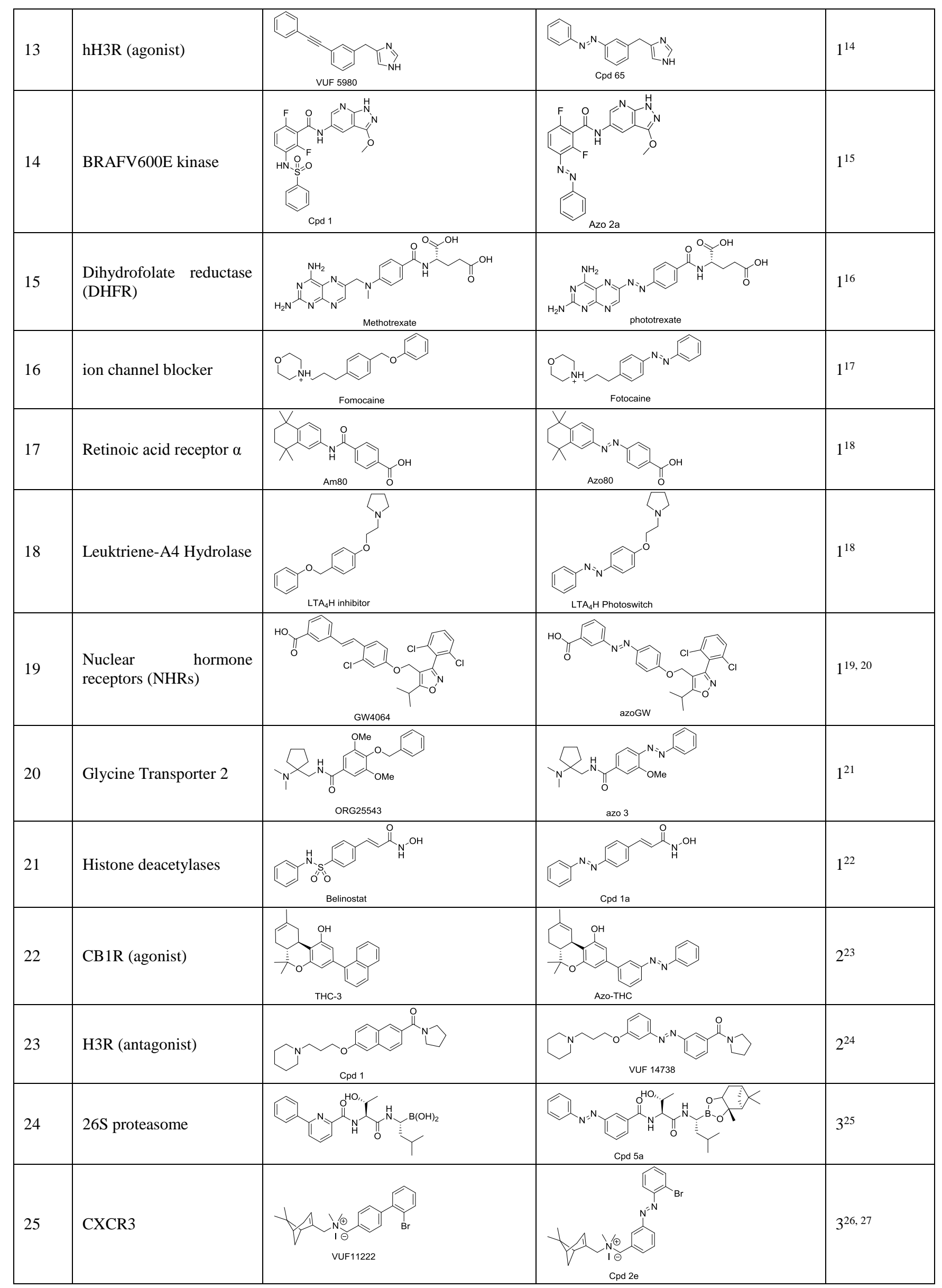




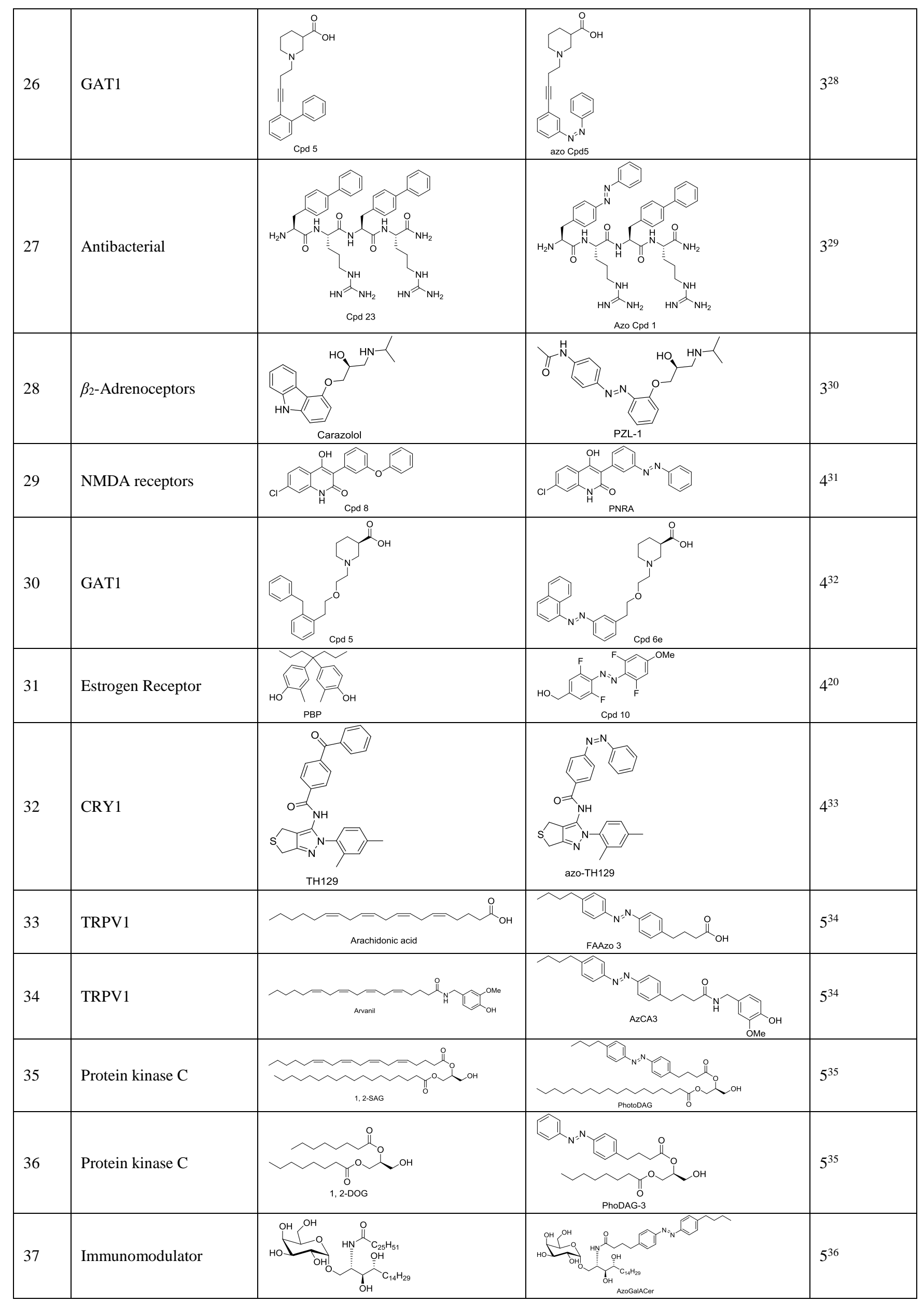




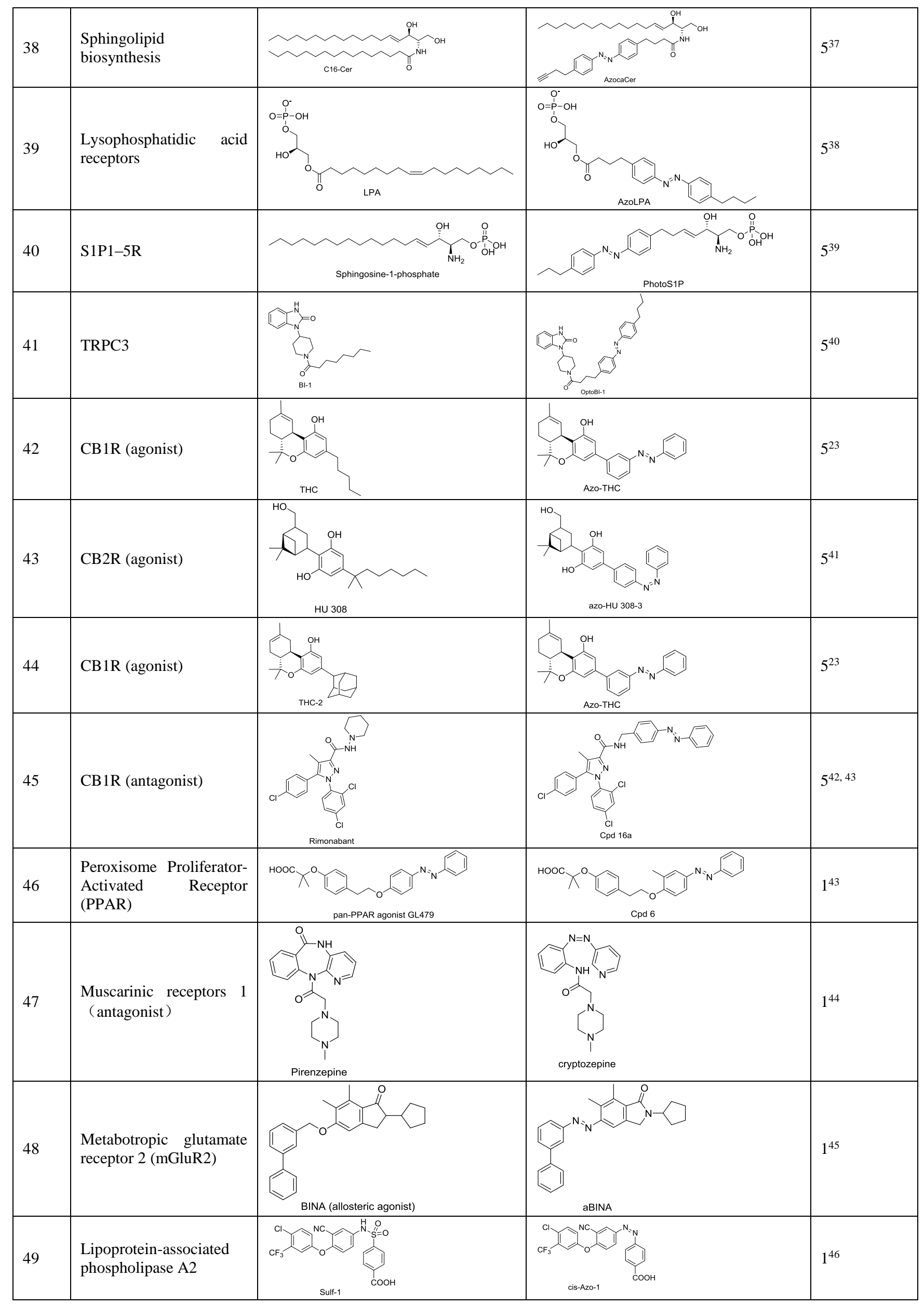




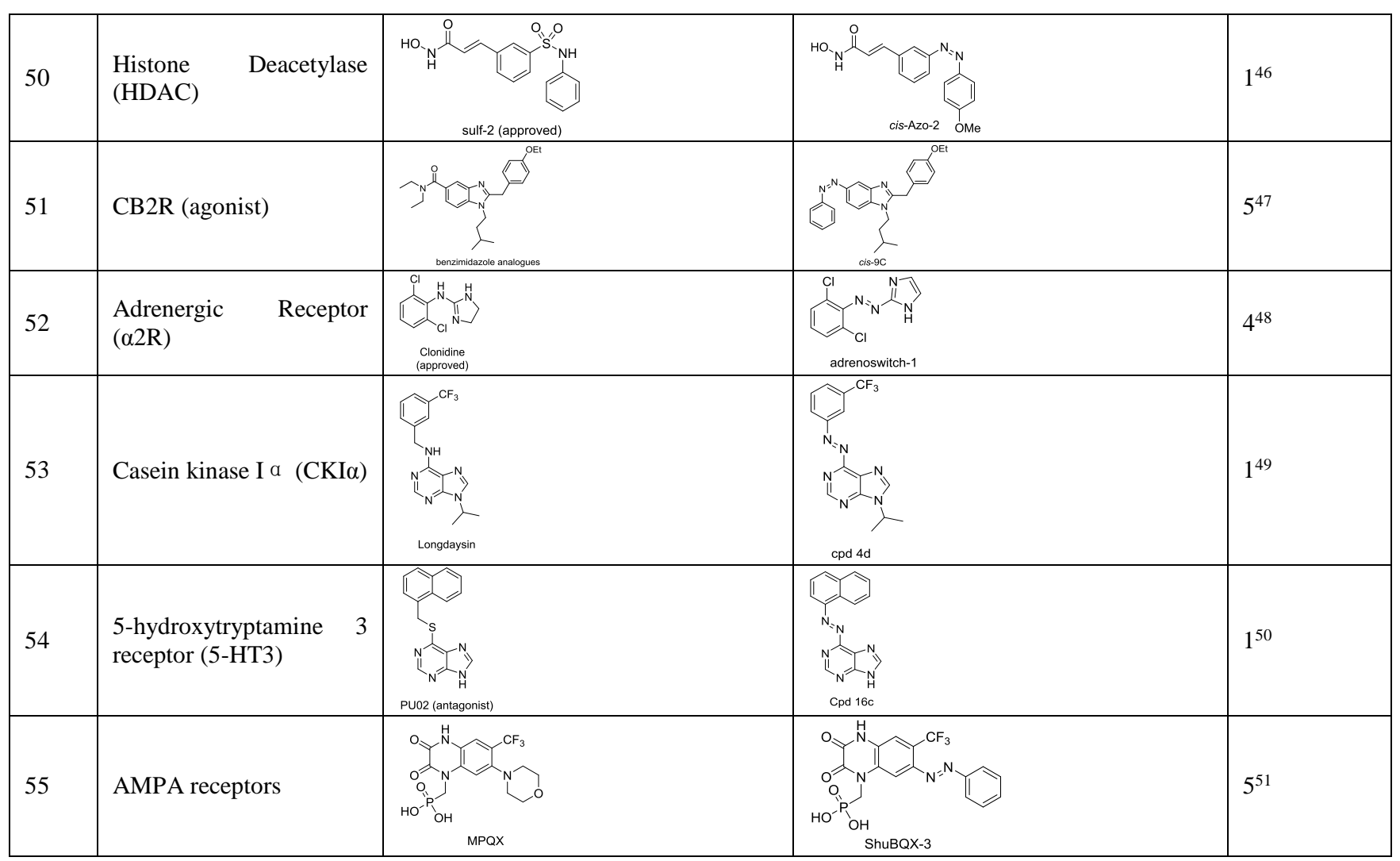

${ }^{a}$ Note: The listed photoswitchable ligands are diffusible and non-covalent that mainly targeting GPCRs, enzyme, transporter/pump, cytoskeleton and ligand-gated ion channel. The ligands obtained by azoextension strategies were omitted. 
Table S2. Overview of ligand statistics from different database.

\begin{tabular}{|c|c|c|c|c|c|c|}
\hline & & Class 1 & Class 2 & Class 3 & Class 4 & Class 5 \\
\hline Database & Total No. & & & & & $\widehat{N_{n}}$ \\
\hline PDB & 26392 & 787 & 601 & 940 & 761 & 165 \\
\hline DrugBank & 9922 & 275 & 190 & 181 & 298 & 51 \\
\hline
\end{tabular}




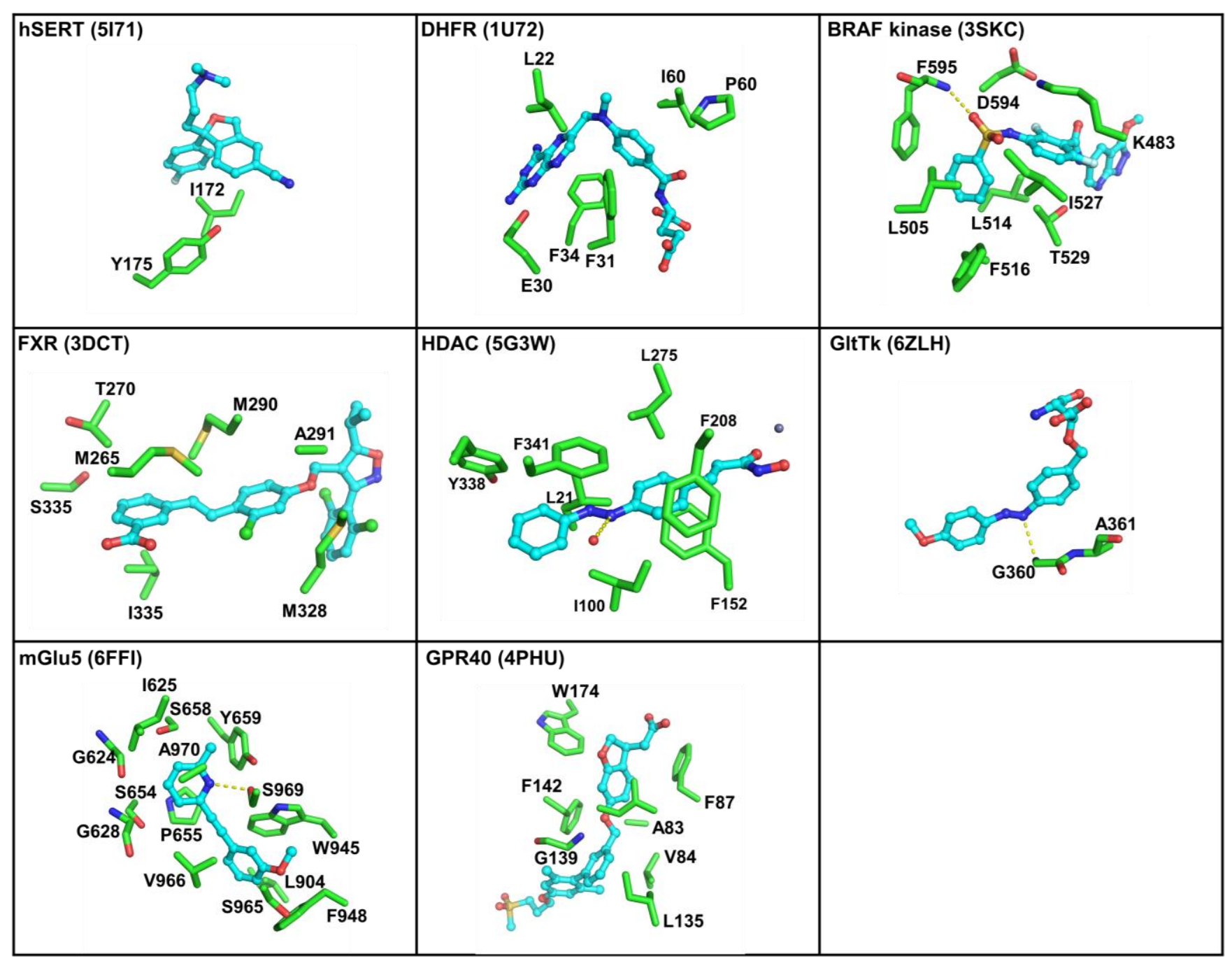

Figure S1. Residue distribution within the range of $4 \AA$ of typical azologization fragments (Class 1) or azobenzene based on the resolved structure. The structure was download from Protein Data Bank (PDB) database (https://www.rcsb.org/) $)^{52}$. The fragment in $3 \mathrm{SKC}^{53}, 5 \mathrm{G} 3 \mathrm{~W}^{22}$, $6 \mathrm{ZLH}^{6}, 6 \mathrm{FFI}^{54}$ involved the formation of hydrogen bond, while $5 \mathrm{I}^{2} 1^{55}, 1 \mathrm{U}^{56} 2^{56}, 3 \mathrm{DCT}^{57}, 4 \mathrm{PHU}^{58}$ were not involved. 


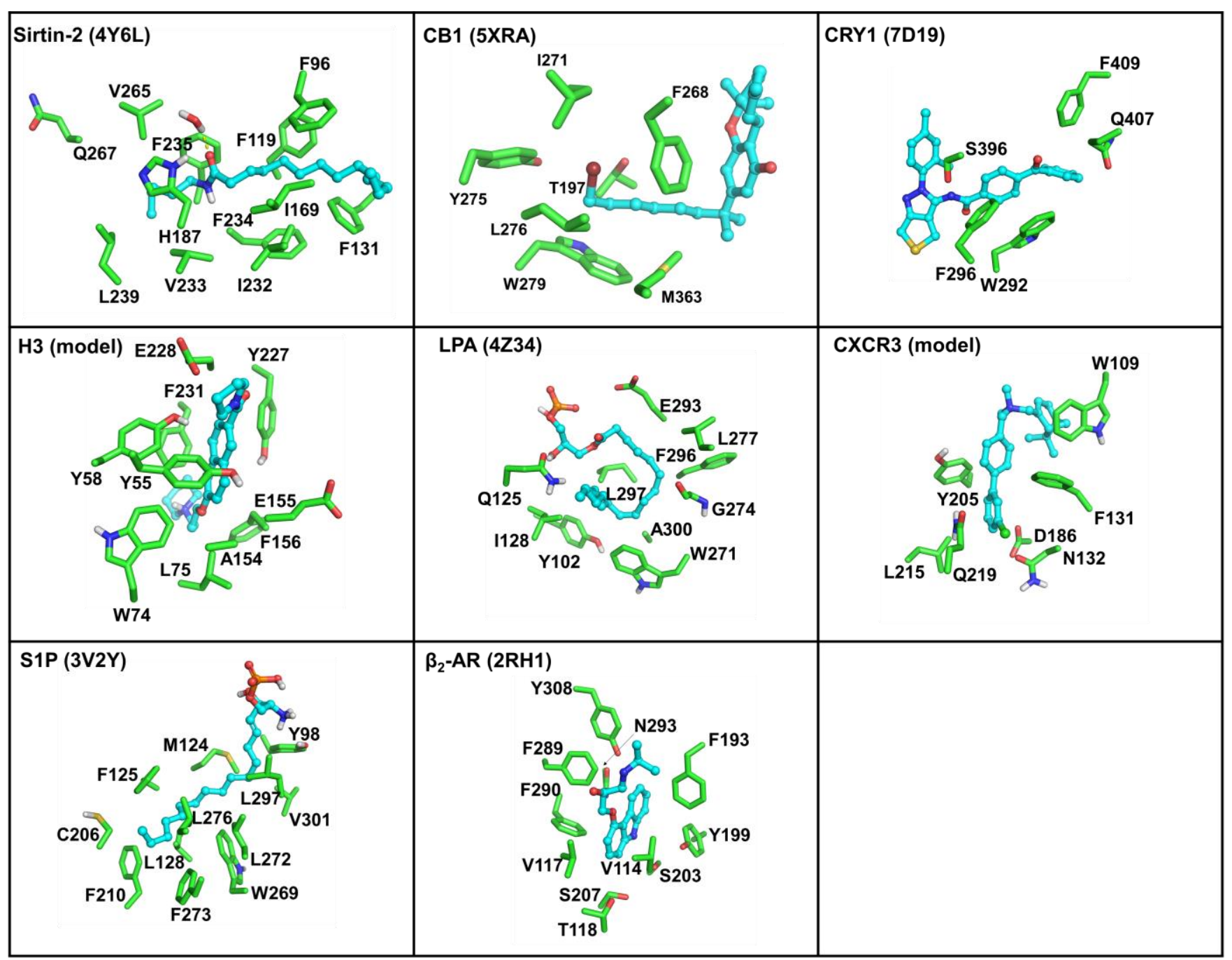

Figure S2. Residue distribution within the range of $4 \AA$ of atypical azologization fragments (Class 2-5). Homology models of H3R and CXCR3 were based on crystal structure of M4 muscarinic acetylcholine receptor $\left(5 \mathrm{DSG}^{59}\right)$ and $\mathrm{C}-\mathrm{X}$-C chemokine receptor type $4\left(3 \mathrm{OE} 8^{60}\right)$, respectively. Binding mode of parent ligand in $4 \mathrm{Y}_{6} \mathrm{~L}^{61}, 4 \mathrm{Z} 34^{62}, 3 \mathrm{~V} 2 \mathrm{Y}^{63}, \mathrm{H} 3 \mathrm{R}$ and CXCR3 were the docking results. 5XRA ${ }^{64}, 7 \mathrm{D} 19^{33}$ and $2 \mathrm{RH}^{65}{ }^{6}$ were the crystal structure. Only the atypical fragment in $4 \mathrm{Y} 6 \mathrm{~L}$ was involved the formation of hydrogen bond. 

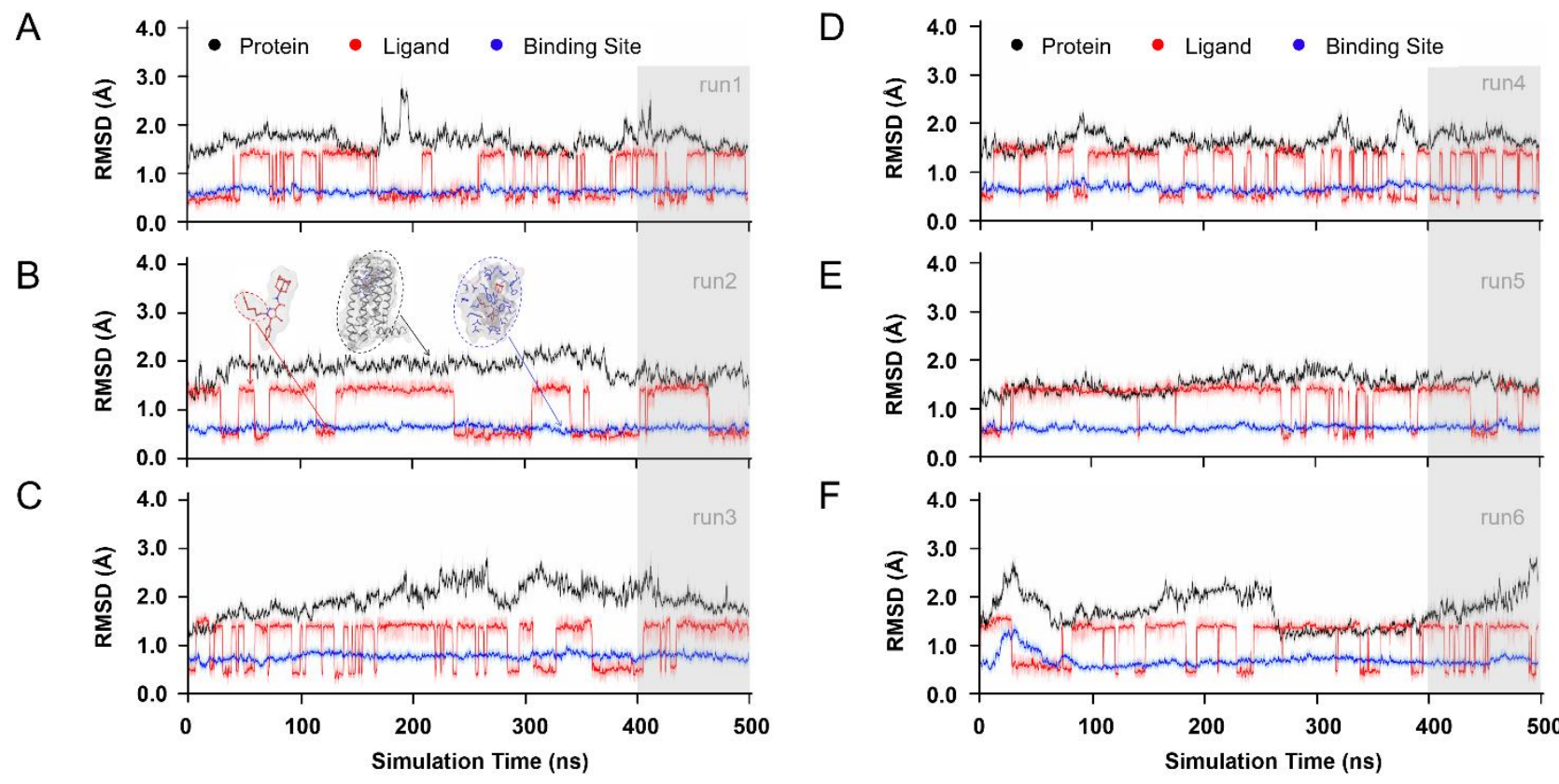

$\mathrm{E}$

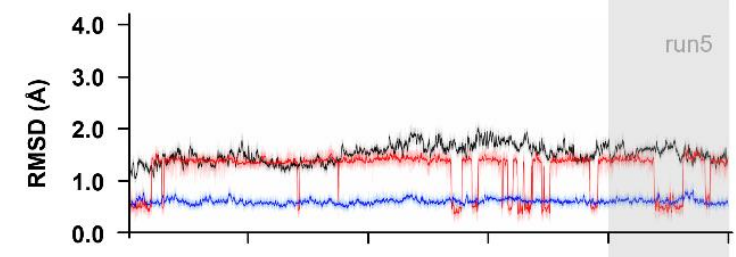

F

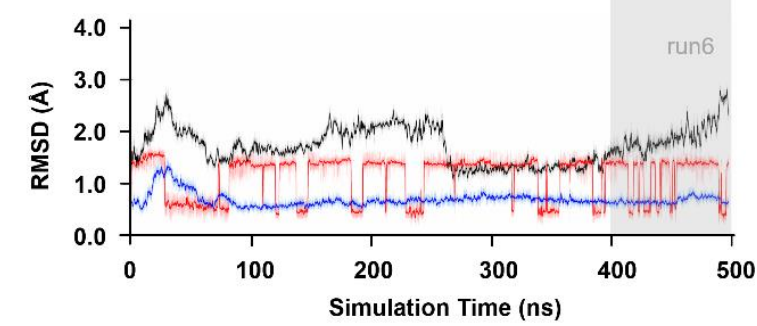

Figure S3. The RMSD values of protein (in dark), ligand (in red) and binding site (in blue) of the studied AM10257-CB2 system against the $500 \mathrm{~ns}$ simulation time for six times (A)-(F). Among them, the last $100 \mathrm{~ns}$ trajectories highlighted in grey background were utilized for the further energy calculation. 


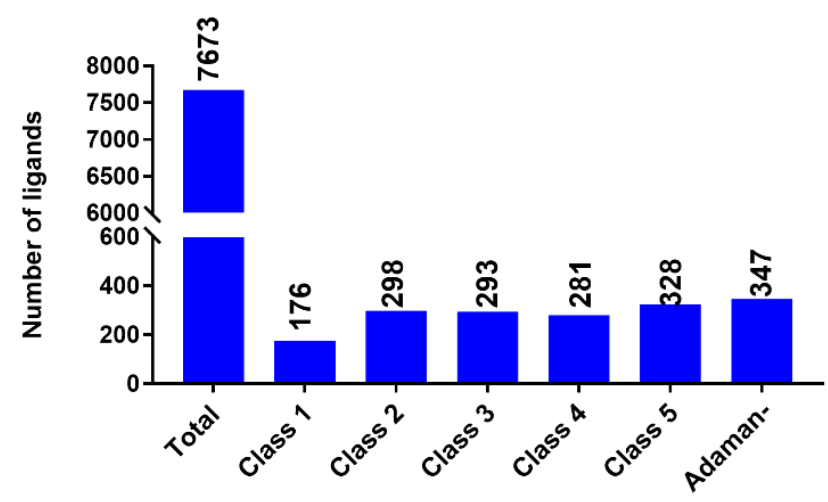

Figure S4. Overview of ligand statistic for CB2. Ligands containing at least one type of fragment from reported CB2 ligands database were counted. Representative fragments were selected for each class. 
<smiles>CCCCCn1cc(C(=O)NC23CC4CC(CC(C4)C2)C3)c(=O)c2ccccc21</smiles>

Cpd 28<smiles>COc1ccccc1-c1cc(C(=O)NCC23CC4CC(CC(C4)C2)C3)no1</smiles>

Cpd 47<smiles>Cc1ccc(C(=O)NCC23CC4CC(CC(C4)C2)C3)cc1S(=O)(=O)N1CCOCC1</smiles>

Cpd 31<smiles>CC1=CC[C@H]2c3c(O)cc(C45CC6CC(CC(C6)C4)C5)cc3OC(C)(C)[C@H]2C1</smiles>

AM411<smiles>C=CCn1cc(C(=O)C(=O)NC23CC4CC(CC(C4)C2)C3)c2cc(-c3ccco3)ccc21</smiles>

\section{Cpd 10a}

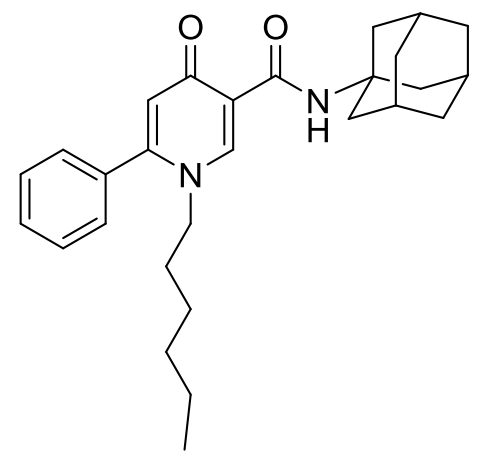

Cpd 25

Figure S5. Representative chemical ligands with adamantly group which would be potentially transformed to azobenzene unit for reported

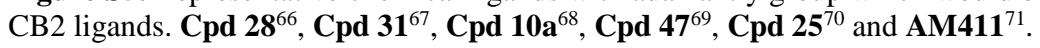


6
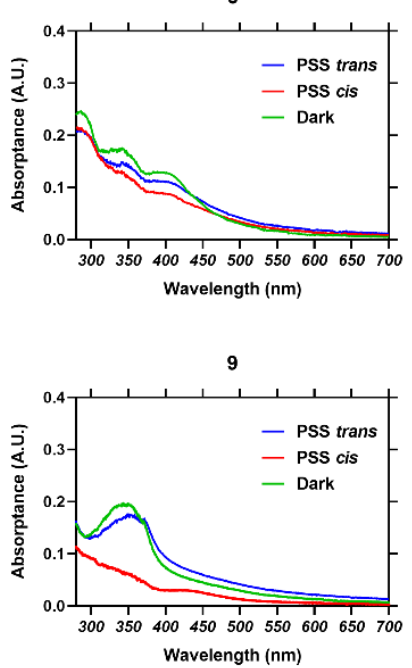

12
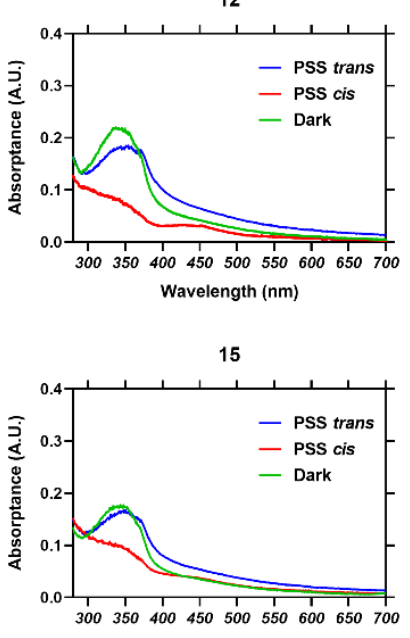

Wavelength $(\mathrm{nm})$
7
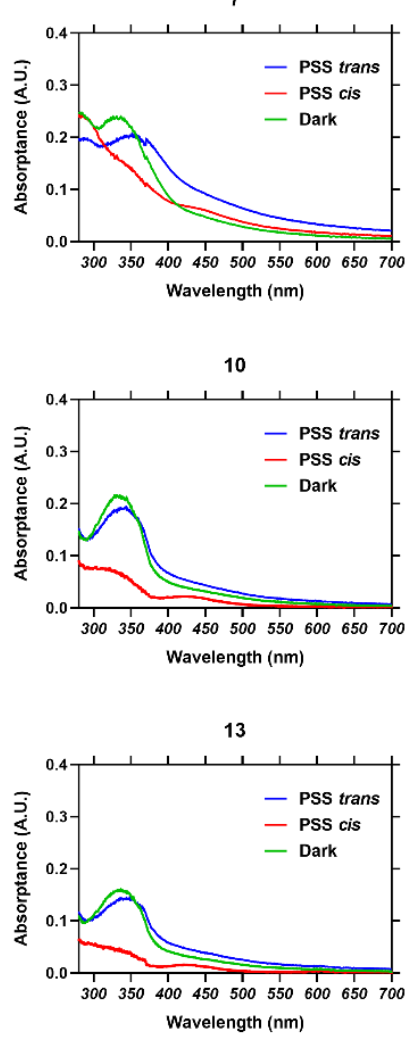

16

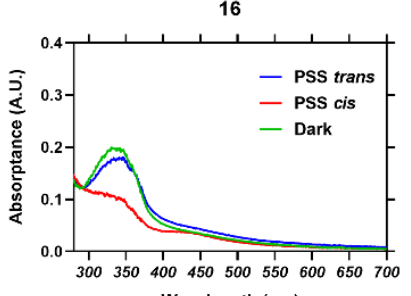

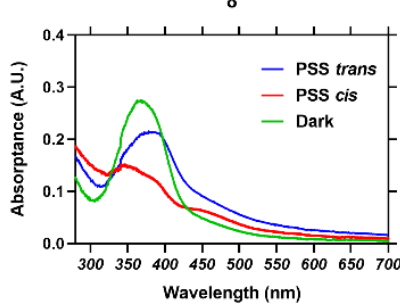
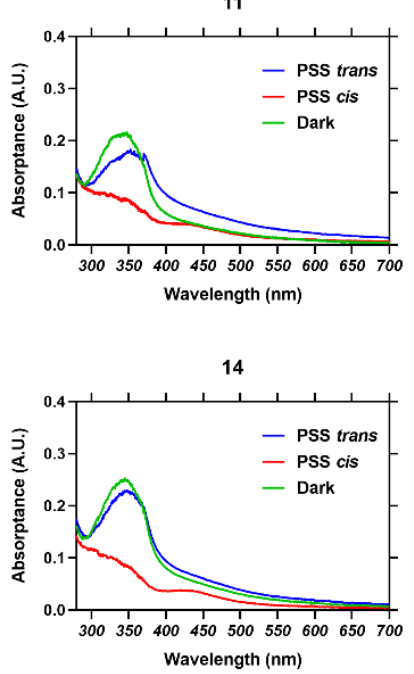

17

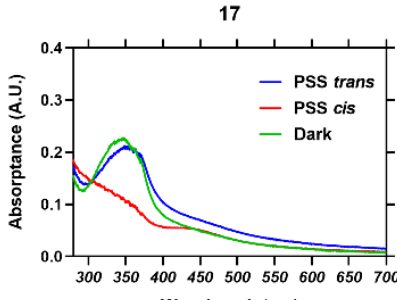

Figure S6. UV-vis spectra for photoswitchable ligands at $12.5 \mu \mathrm{M}$ concentration in HBSS. PSS cis represents a sample which has been illuminated for $150 \mathrm{~s}$ using $365 \pm 20 \mathrm{~nm}$ light (red). PSS trans represents subsequent illumination for $150 \mathrm{~s}$ using $435 \pm 10 \mathrm{~nm}$ (blue). Dark represents the pure $(E)$-form absorption which were obtained by heating pretreatment (green).

Table S3. The maximum absorption wavelength for trans and cis isomers in HBSS at $12.5 \mu \mathrm{M}$.

\begin{tabular}{ccccccccccccc}
\hline AzoLigs & $\mathbf{6}$ & $\mathbf{7}$ & $\mathbf{8}$ & $\mathbf{9}$ & $\mathbf{1 0}$ & $\mathbf{1 1}$ & $\mathbf{1 2}$ & $\mathbf{1 3}$ & $\mathbf{1 4}$ & $\mathbf{1 5}$ & $\mathbf{1 6}$ & $\mathbf{1 7}$ \\
\hline $\begin{array}{c}\lambda_{\max } \\
(\text { trans })\end{array}$ & $342 / 399$ & 333 & 368 & 349 & 334 & 341 & 343 & 337 & 344 & 344 & 337 & 346 \\
\hline $\begin{array}{c}\lambda_{\max } \\
(\text { cis })\end{array}$ & 469 & 445 & 455 & 436 & 433 & 436 & 447 & 433 & 435 & 437 & 434 & 442 \\
\hline
\end{tabular}


6
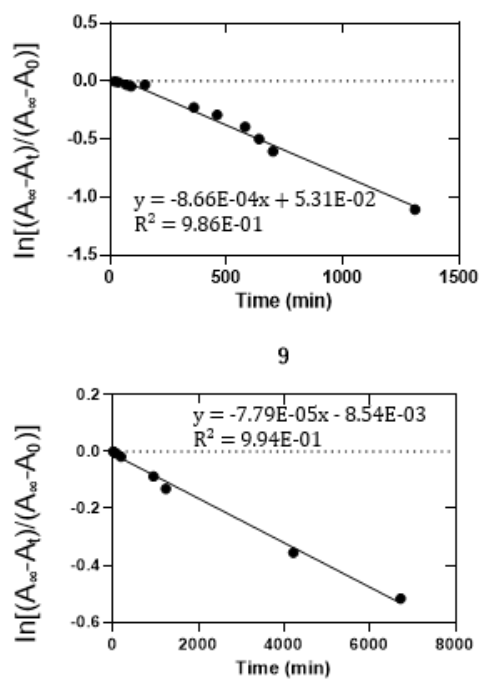

12

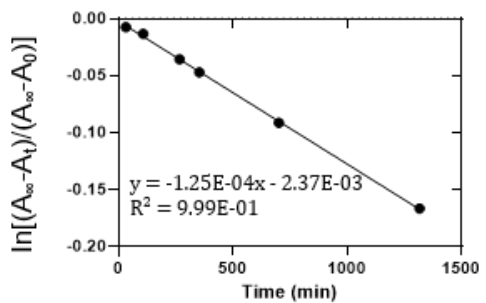

15

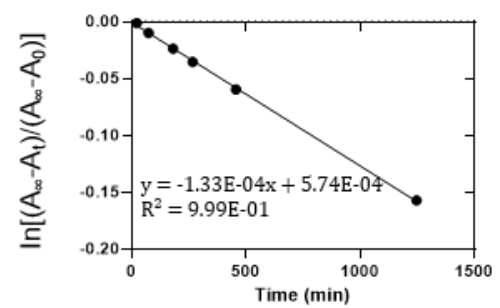

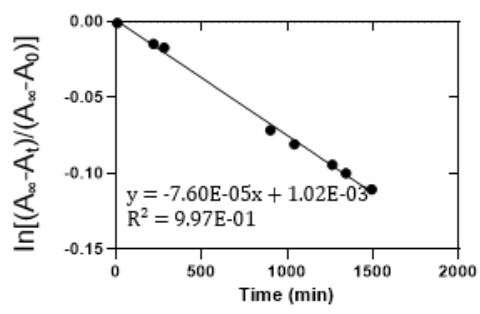

10
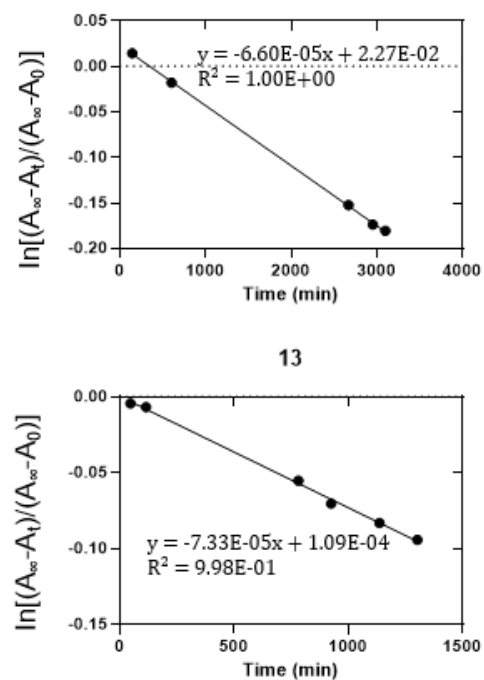

16

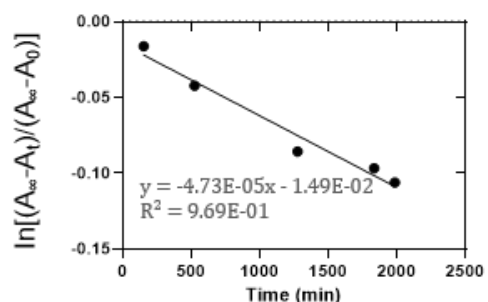

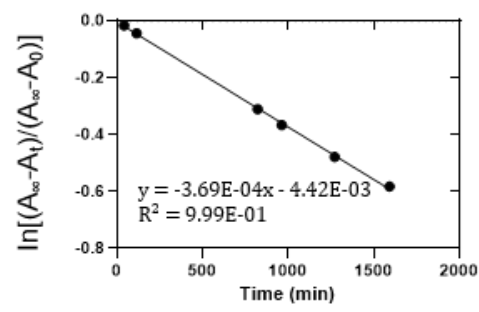

11

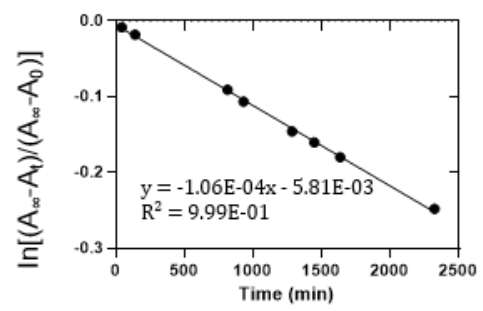

14
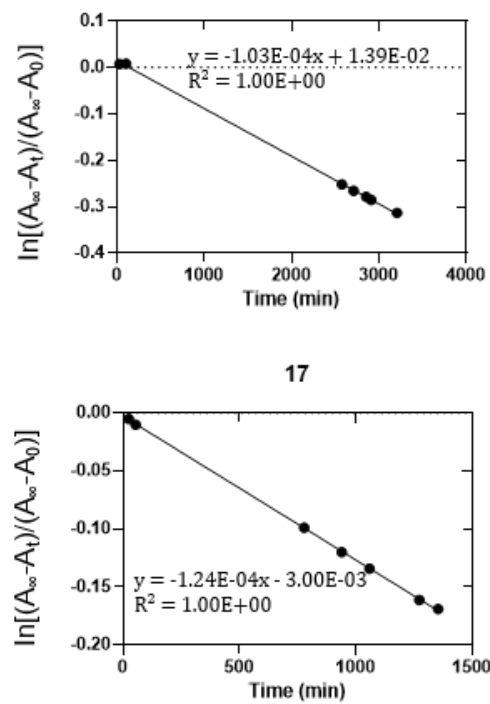

Figure S7. Thermal isomerization kinetics for each compound (AzoLig 6-17) under room temperature as determined by UV-Vis spectra in DMSO. Regression equation and $\mathrm{R}^{2}$ (regression coefficient) were displayed at top-right. The absolute value of slope was the cis-trans isomerization rate constant.

Table S4. Half-life for cis-form of ligands under room temperature in DMSO.

\begin{tabular}{ccccccccccccc}
\hline AzoLig & $\mathbf{6}$ & $\mathbf{7}$ & $\mathbf{8}$ & $\mathbf{9}$ & $\mathbf{1 0}$ & $\mathbf{1 1}$ & $\mathbf{1 2}$ & $\mathbf{1 3}$ & $\mathbf{1 4}$ & $\mathbf{1 5}$ & $\mathbf{1 6}$ & $\mathbf{1 7}$ \\
\hline $\mathbf{t}_{\mathbf{1} / 2}(\mathbf{h})$ & 13.3 & 152.0 & 31.3 & 136.3 & 174.9 & 109.0 & 92.4 & 157.6 & 112.3 & 87.9 & 244.4 & 93.3 \\
\hline
\end{tabular}


Table S5. Ratio of cis-AzoLig $9(2.5 \mathrm{mM})$ increases with extended illumination under room temperature as determined by ${ }^{1} \mathrm{H}$ NMR in DMSO-d6. Irradiation treatment performed in the quartz cuvette with LED (Benstartech, Cat.\# BS-SD-UV-01, Size: $49 * 72$ mm, Power: 4 W, Wavelength: $365 \pm 20 \mathrm{~nm}$ ).

\begin{tabular}{cccccc}
\hline Illumination time (s) & 0 & 150 & 300 & 600 & 900 \\
\hline Ratio of cis-form & $<5 \%$ & $22.5 \%$ & $53.9 \%$ & $86.4 \%$ & $93.3 \%$ \\
\hline
\end{tabular}


<PDA Ch1 254 nm>

mAt

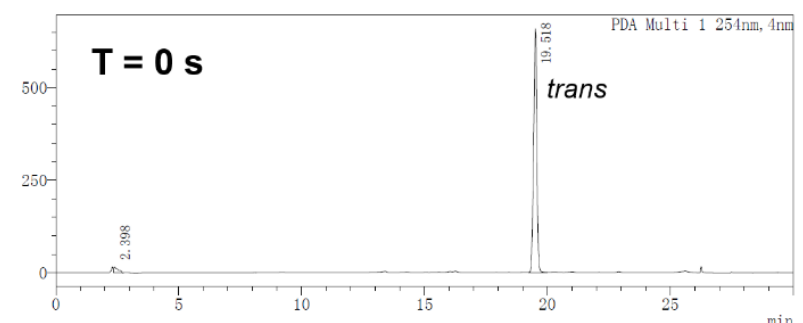

PDA Ch1 254nm

\begin{tabular}{|c|c|c|c|c|c|}
\hline Peak\# & Ret. Time $(\mathrm{min})$ & Height $(\mathrm{mAU})$ & Height $\%$ & Area(mAU*s) & Area\% \\
\hline 1 & 2.398 & 15339 & 2.276 & 203119 & 3.243 \\
\hline 2 & 19.518 & 658552 & 97.724 & 6060995 & 96.757 \\
\hline Sum & & 673891 & 100.000 & 6264114 & 100.000 \\
\hline
\end{tabular}

<PDA Ch1 254 nm>

$\mathrm{mAL}$

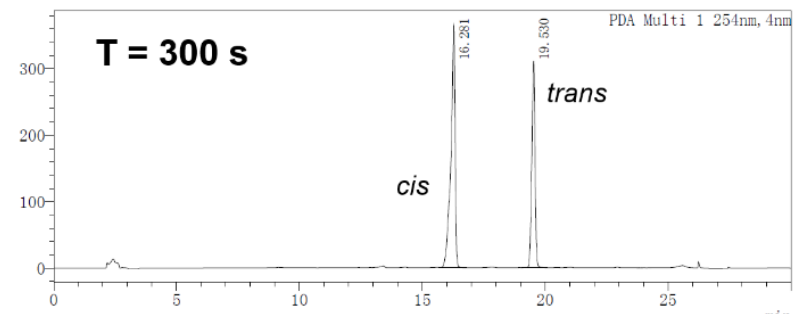

PDA Ch1 254nm

\begin{tabular}{|c|c|c|c|c|c|}
\hline PDA Ch\# & Ret. Time (min) & Height(mAU) & Height $\%$ & Area(mAU*s) & Area\% \\
\hline 1 & 16.281 & 366561 & 54.081 & 4361905 & 60.546 \\
\hline 2 & 19.530 & 311235 & 45.919 & 2842376 & 39.454 \\
\hline Sum & & 677796 & 100.000 & 7204282 & 100.000 \\
\hline
\end{tabular}

\section{<PDA Ch1 254 nm>}

mAU

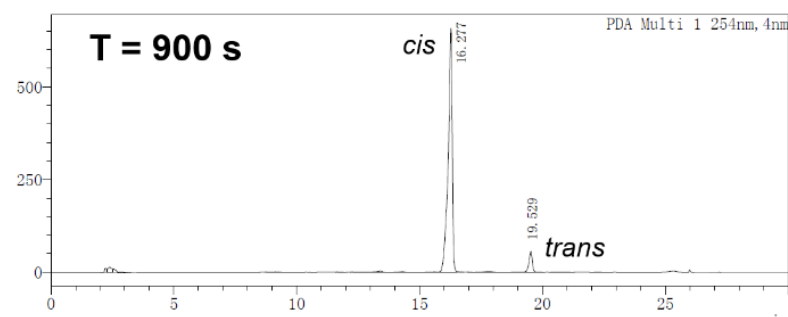

PDA Ch1 254nm

\begin{tabular}{|c|c|c|c|c|c|}
\hline Peak\# & Ret. Time (min) & Height(mAU) & Height\% & Area(mAU*s) & Area\% \\
\hline 1 & 16.277 & 657038 & 92.158 & 7546095 & 93.539 \\
\hline 2 & 19.529 & 55910 & 7.842 & 521254 & 6.461 \\
\hline Sum & & 712948 & 100.000 & 8067349 & 100.000 \\
\hline
\end{tabular}

<PDA Ch1 $254 \mathrm{~nm}>$

$\mathrm{mAl}$

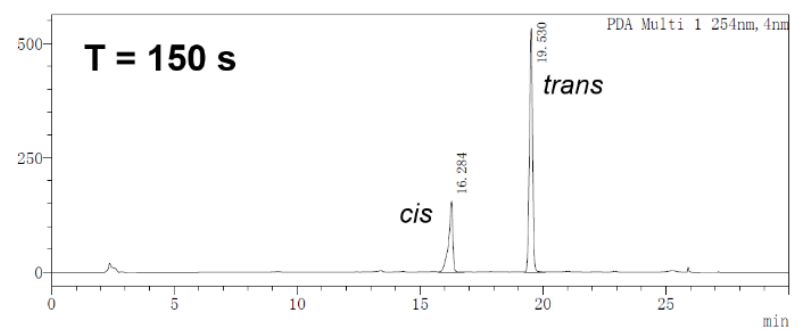

DA Ch1 254nm

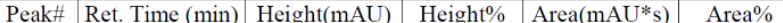

\begin{tabular}{|c|c|c|c|c|c|}
\hline Peak\# & Ret. Time $(\mathrm{min})$ & Height $(\mathrm{mAU})$ & Height $\%$ & Area $(\mathrm{mAU} * \mathrm{~s})$ & Area $\%$ \\
\hline 1 & 16.284 & 154063 & 22.450 & 1823116 & 27.641 \\
\hline 2 & 19.530 & 532202 & 77.550 & 4772589 & 72.359 \\
\hline Sum & & 686266 & 100.000 & 6595704 & 100.000 \\
\hline
\end{tabular}

<PDA Ch1 254 nm>

$\mathrm{mAL}$

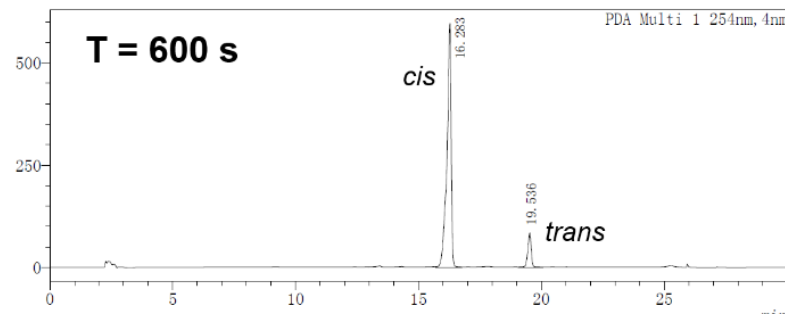

PDA Ch1 254nm

\begin{tabular}{|c|c|c|c|c|c|}
\hline Peak\# & Ret. Time (min) & Height $(\mathrm{mAU})$ & Height $\%$ & Area(mAU*s) & Area\% \\
\hline 1 & 16.283 & 595589 & 87.685 & 7049625 & 89.958 \\
\hline 2 & 19.536 & 83645 & 12.315 & 786960 & 10.042 \\
\hline Sum & & 679234 & 100.000 & 7836584 & 100.000 \\
\hline
\end{tabular}

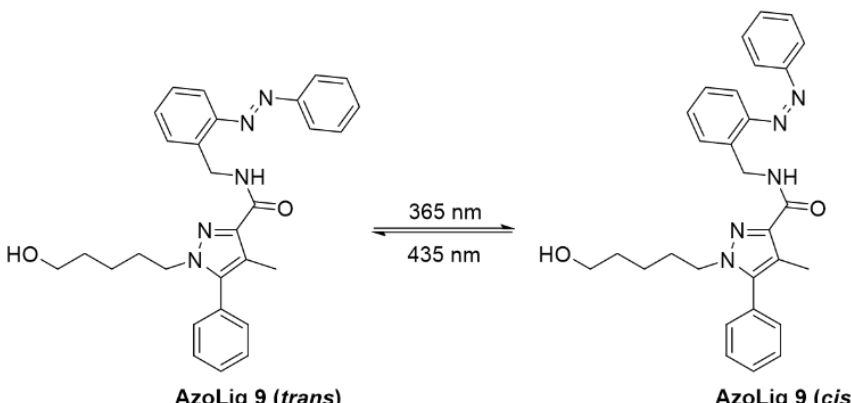

Figure S8. HPLC spectra of AzoLig 9 by illumination for different time at $365 \pm 20$ nm. Illumination time (T) in seconds. Spectra was collected at $2.5 \mathrm{mM}$ in DMSO- $d_{6}$. trans-form could be converted into cis-form within 15 min. Irradiation treatment was described in Table S5.

Table S6. Ratio of cis-AzoLig 9 increases with the extended illumination as determined by HPLC.

\begin{tabular}{cccccc}
\hline Illumination time (s) & 0 & 150 & 300 & 600 & 900 \\
\hline Ratio of cis & $<5 \%$ & $27.6 \%$ & $60.5 \%$ & $90.0 \%$ & $93.5 \%$ \\
\hline
\end{tabular}




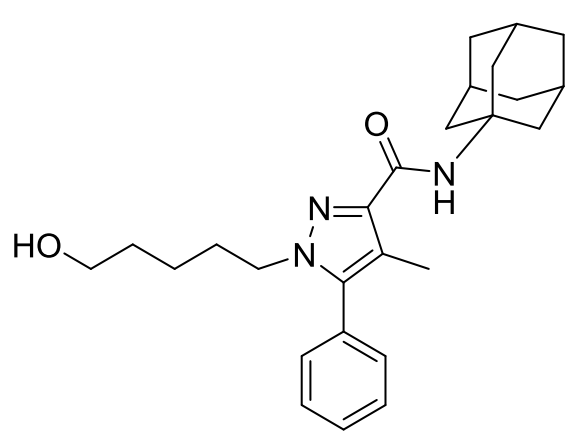

AM10257

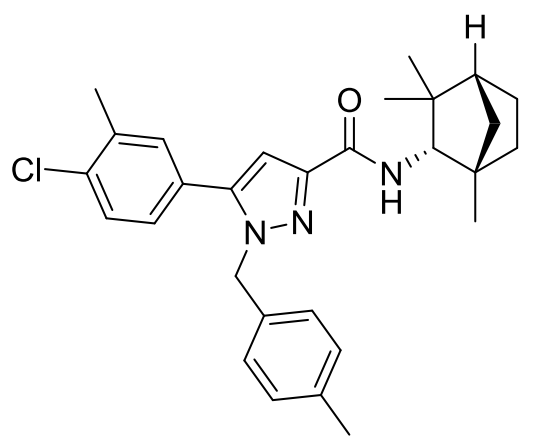

SR144528

Figure S9. Chemical structures of parent ligand AM1025772 and typical CB2 selective antagonist SR144528 ${ }^{73}$.

Table S7. Pharmacological Activity of AM10257 and SR144528 for hCB2R.

\begin{tabular}{ccc}
\hline Cpd. & AM10257 & SR144528 \\
\hline IC $_{50}(\mathrm{nM})^{[\mathrm{a}]}$ & $47 \pm 6$ & $223 \pm 89$ \\
\hline
\end{tabular}

[a] All pharmacology experiments were performed independently in triplicate. 
A

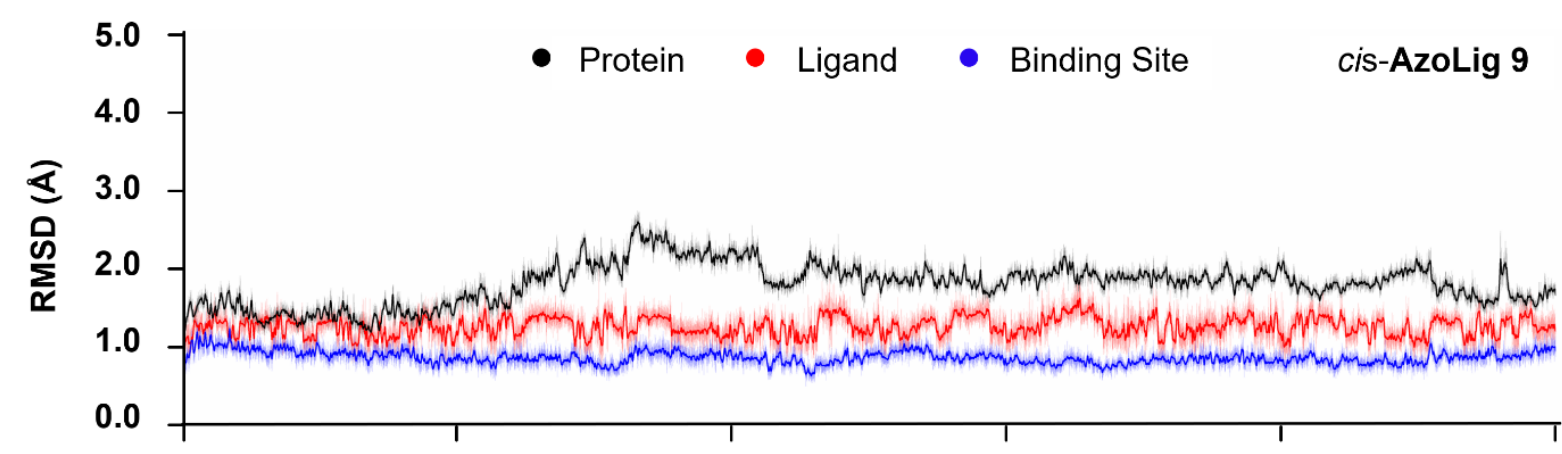

B

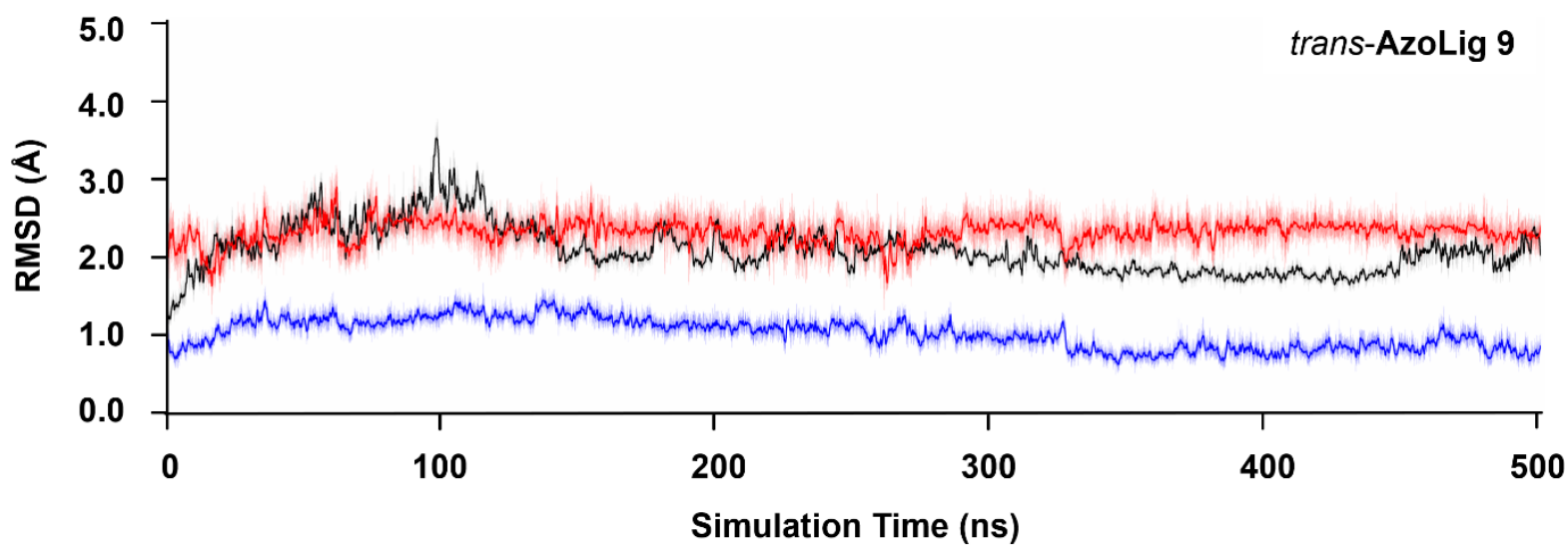

Figure S10. The RMSD values of protein (in dark), ligand (in red) and binding site (in blue) of the studied cis-AzoLig 9-CB2 (A) and transAzoLig 9-CB2 (B) system against the 500 ns simulation time for six times. The representative snapshots for the proposed binding modes of AzoLig 9 were extracted from the last 100 ns trajectories based on the RMSD values of the snapshots shifted from the initial poses. 

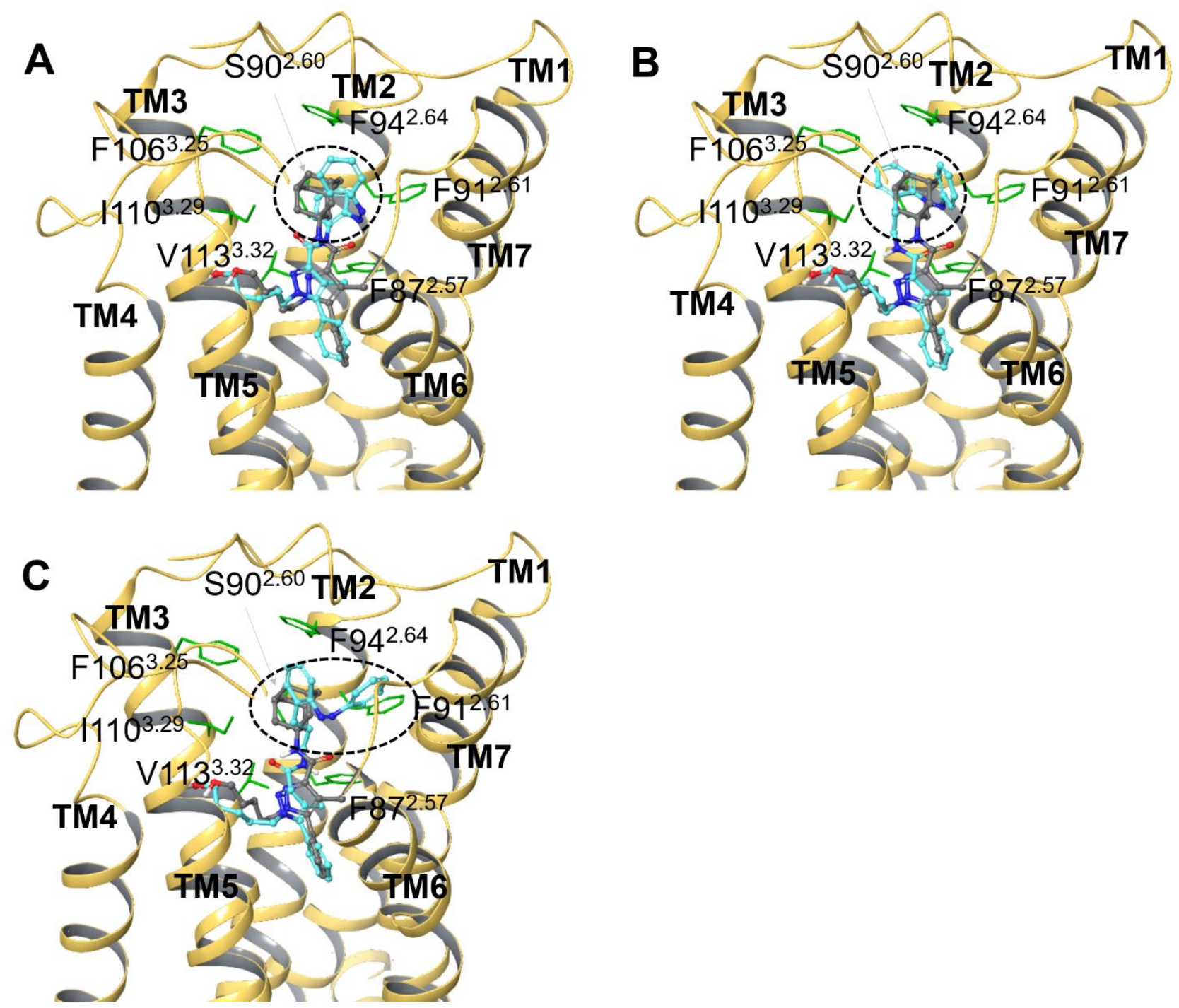

Figure S11. The proposed binding modes of cis-form of ortho-substituted AzoLig 6 (A), 12 (B) and 15 (C) with different spacer length (PDB ID: 5ZTY). The identified key residues in the sub-pocket III were in green stick. The AzoLigs and AM10257 were displayed in cyan and dark ball-and-stick, respectively. Azobenzene moieties within AzoLigs and adamantyl moiety within AM10257 were cycled in dashed lines. 

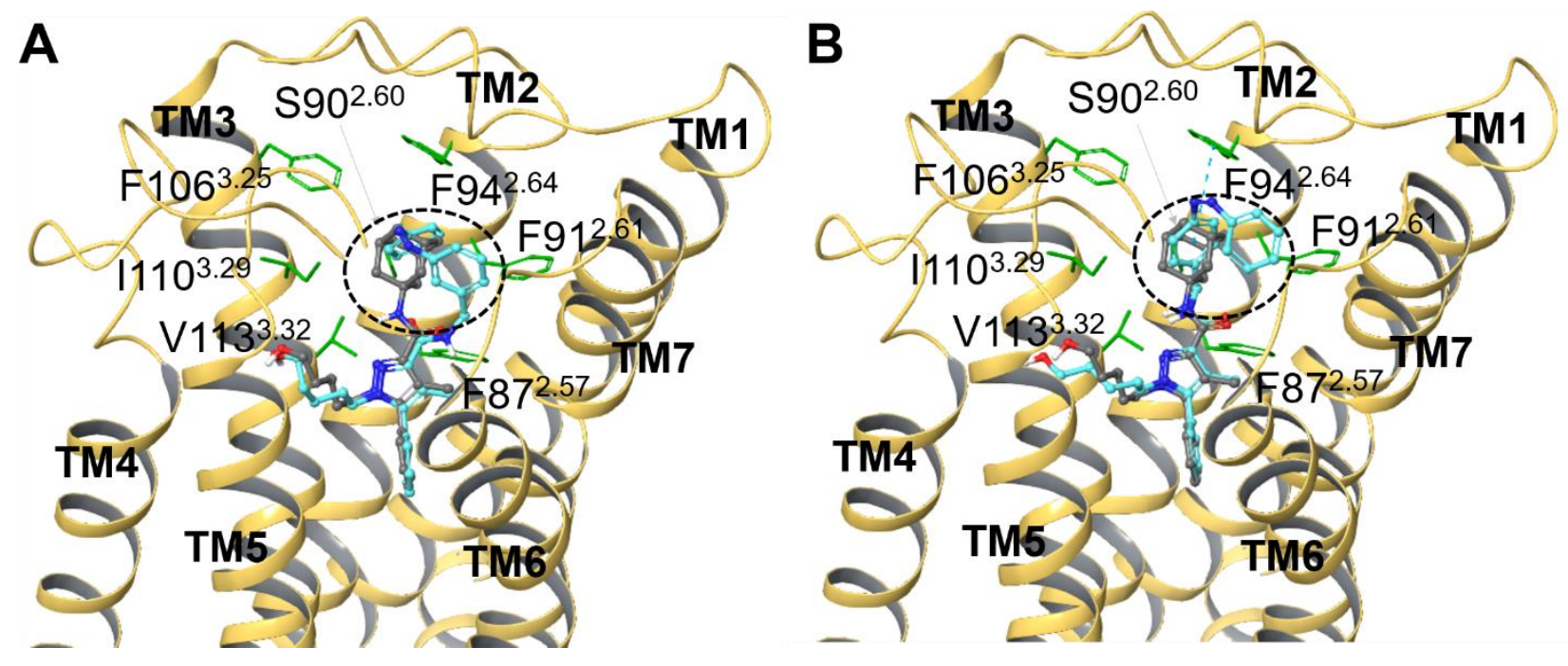

Figure S12. The proposed binding modes of cis-form of AzoLig 10 (A) and 11 (B) with different azo position at fixed spacer length (PDB ID: 5ZTY). The identified key residues in the sub-pocket III were in green stick. The AzoLigs and AM10257 were displayed in cyan and dark ball-and-stick, respectively. Azobenzene moieties within AzoLigs and adamantyl moiety within AM10257 were cycled in dashed lines. 
A

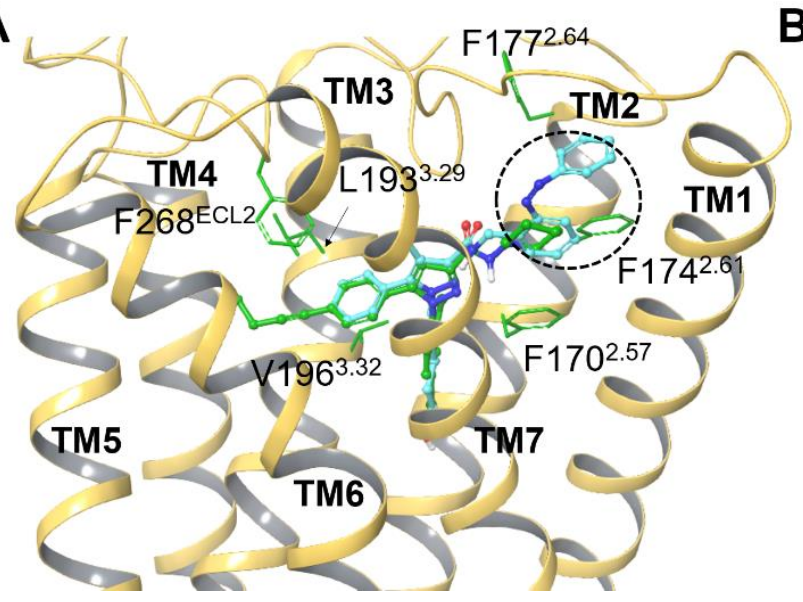

B

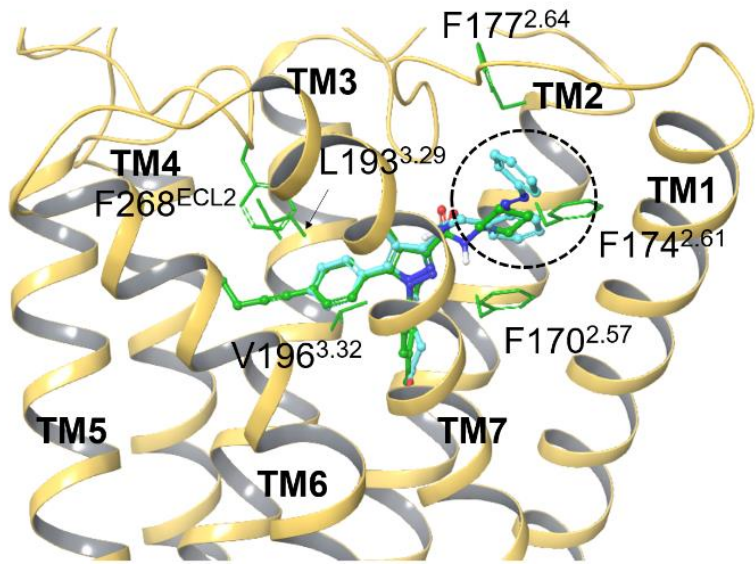

Figure S13. The proposed binding modes of trans-/cis-AzoLig 9 to CB1 (PDB ID: 5TGZ). (A) The binding mode of cis-AzoLig 9 in CB1. (B) The binding mode of trans-AzoLig 9. The protein was displayed in gold cartoon. The aromatic and hydrophobic residues located in the transmembrane II and III were in green stick. AzoLig 9 and AM6538 were displayed in cyan and green ball-and-stick, respectively. Azobenzene moieties within AzoLig 9 and piperidine moiety within AM6538 were cycled in dashed lines. 
NMR spectra of final photoswitchable ligands

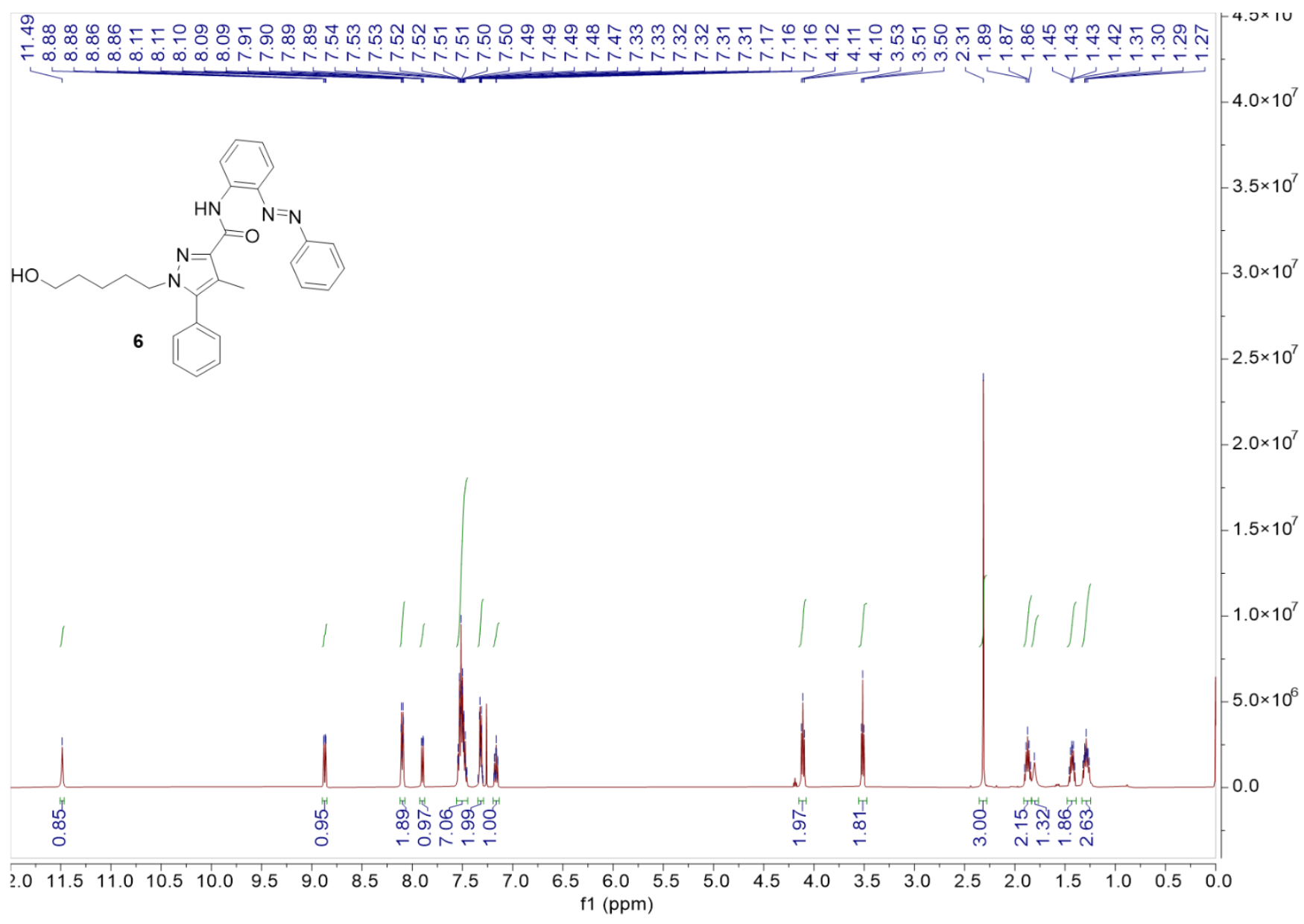




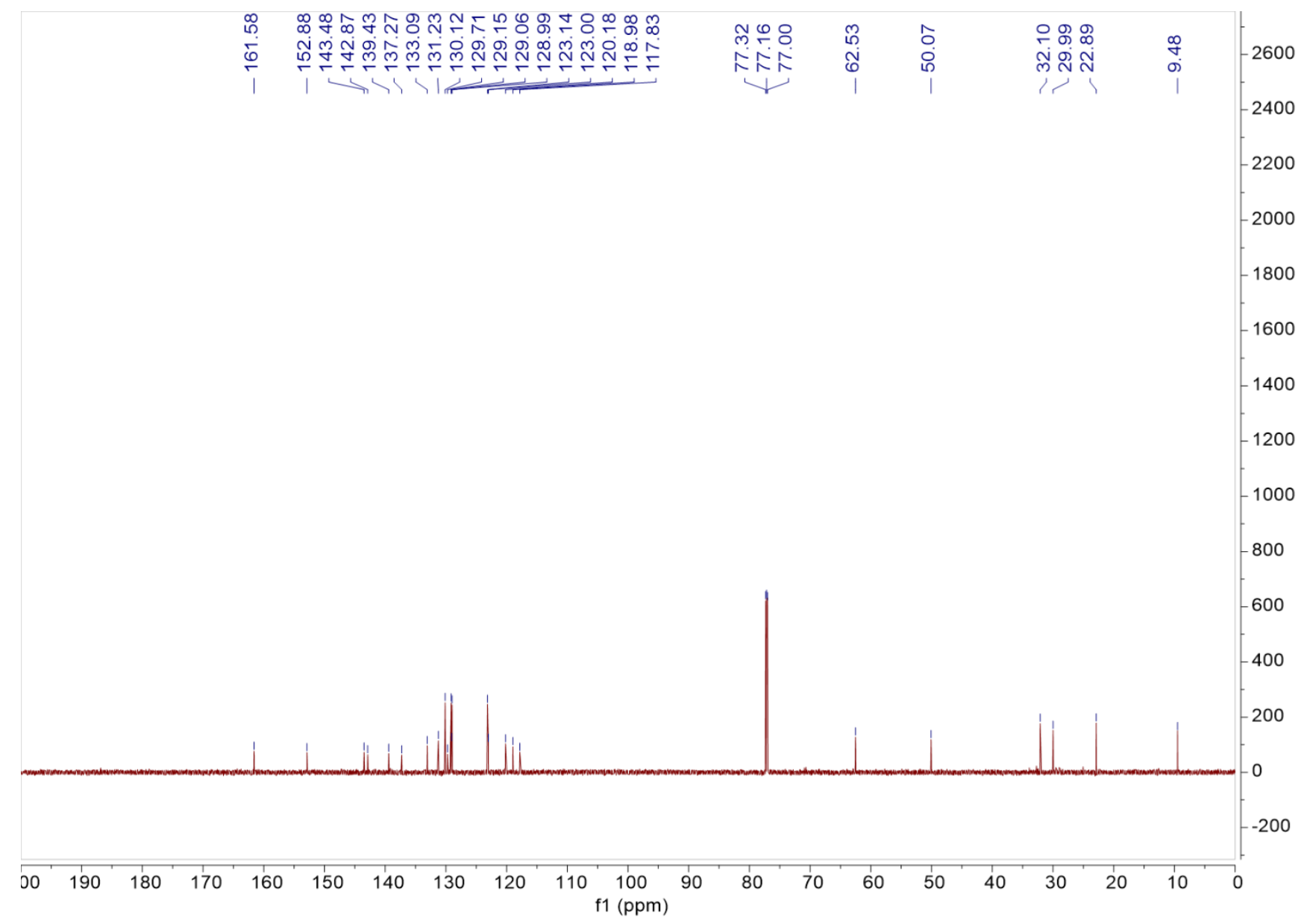



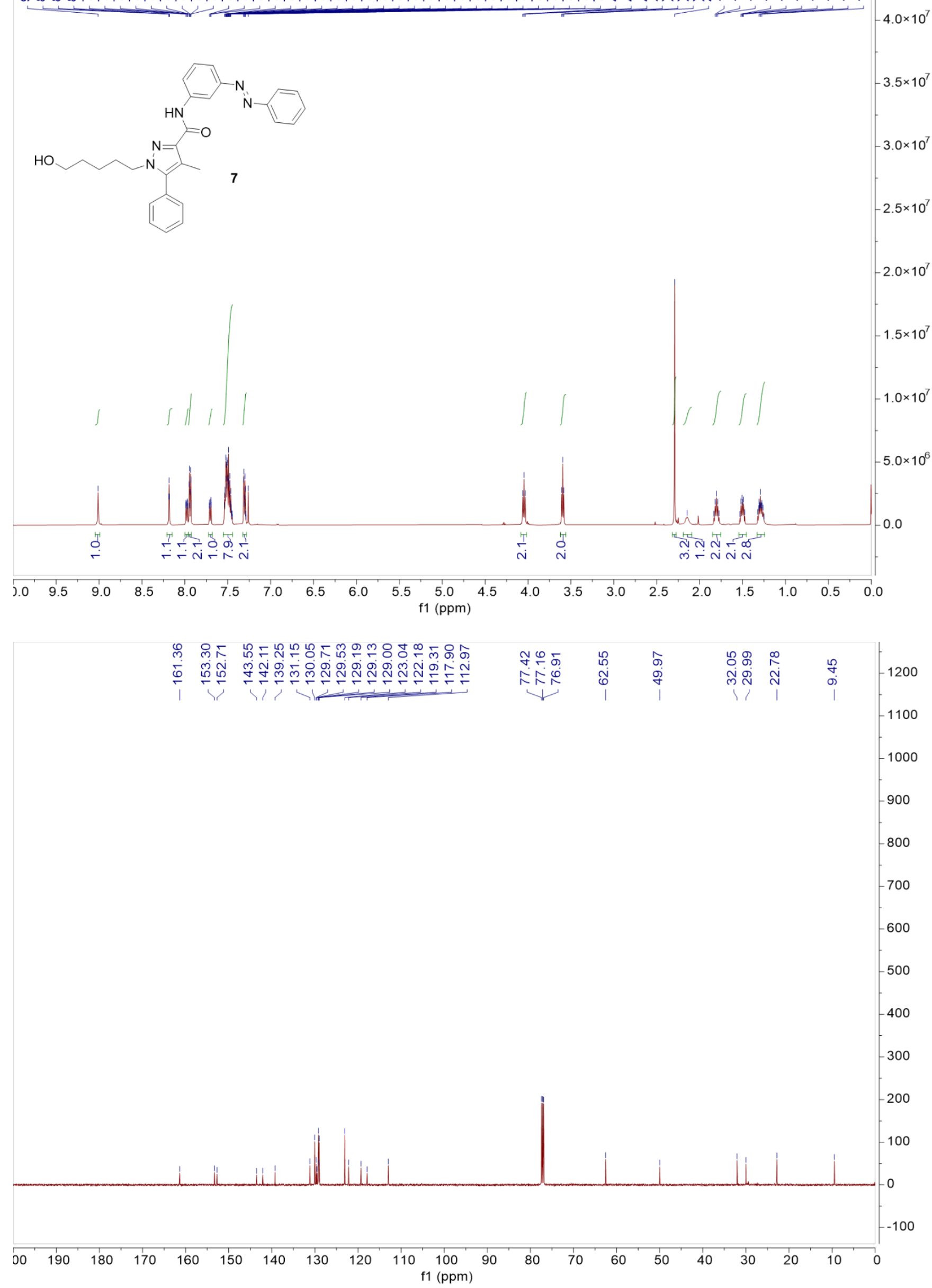

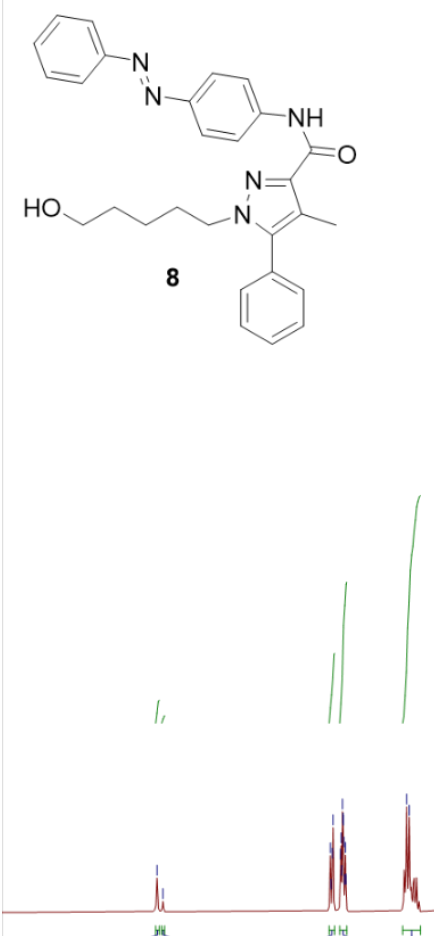

\begin{tabular}{|c|c|c|c|c|c|c|c|c|c|c|c|c|c|c|c|c|c|c|c|}
\hline & $\begin{array}{c}\infty \\
0 \\
0 \\
0 \\
0 \\
0\end{array}$ & & ํํㅇ & 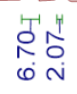 & & & & & & के & $\frac{\text { 'v }}{\text { ì }}$ & 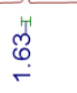 & 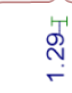 & तु & & 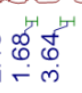 & & & $\begin{array}{l}-0 \\
--20000\end{array}$ \\
\hline 9.5 & 9.0 & 8.5 & 8.0 & 7.5 & 7.0 & 6.5 & 6.0 & 5.5 & $\begin{array}{l}5.0 \\
\text { f1 }(\mathrm{ppm})\end{array}$ & 4.5 & 4.0 & 3.5 & 3.0 & 2.5 & 2.0 & 1.5 & 1.0 & 0.5 & 0.0 \\
\hline
\end{tabular}

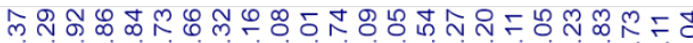

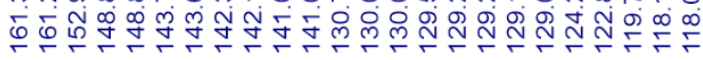

궁요 

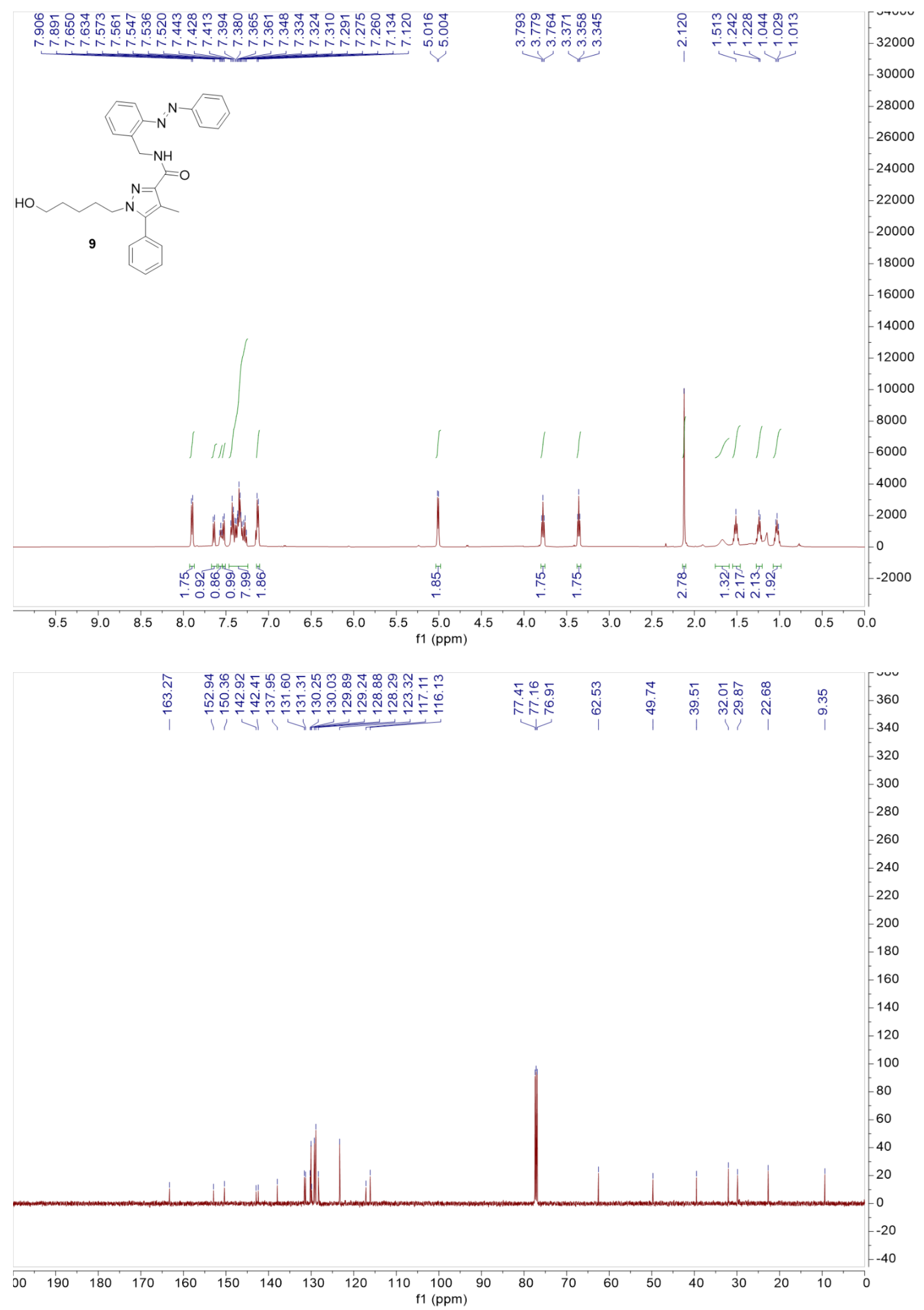
J
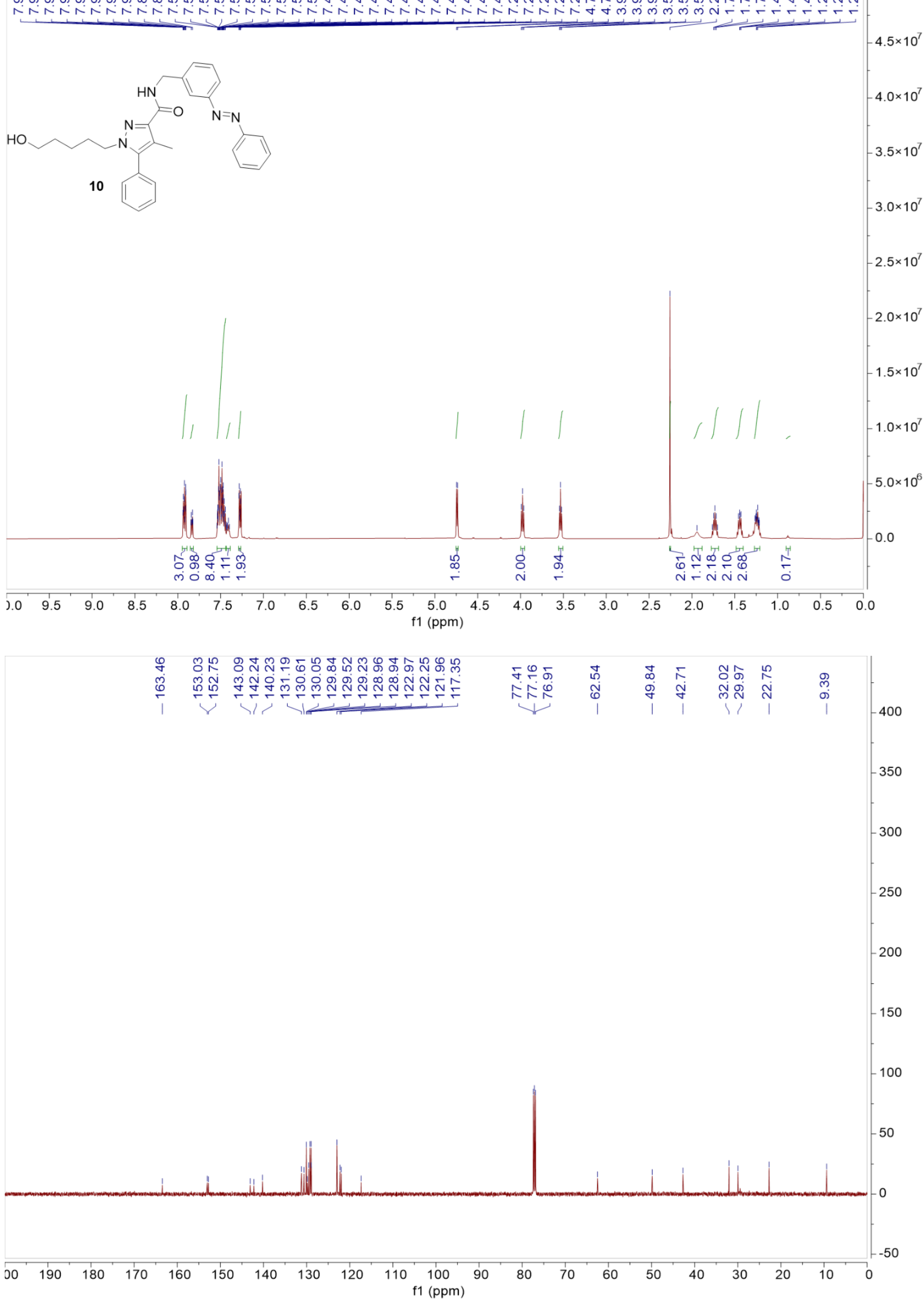


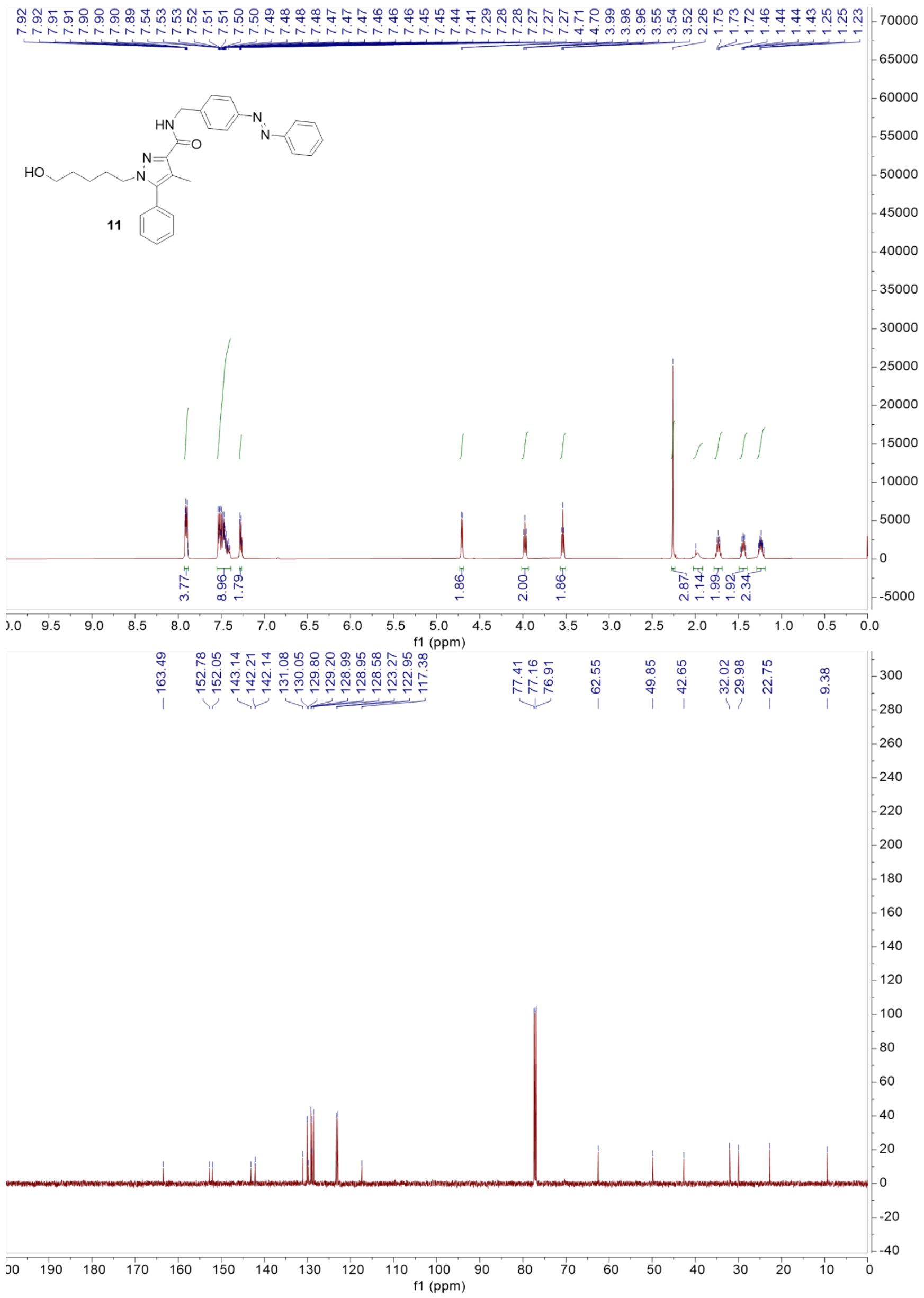



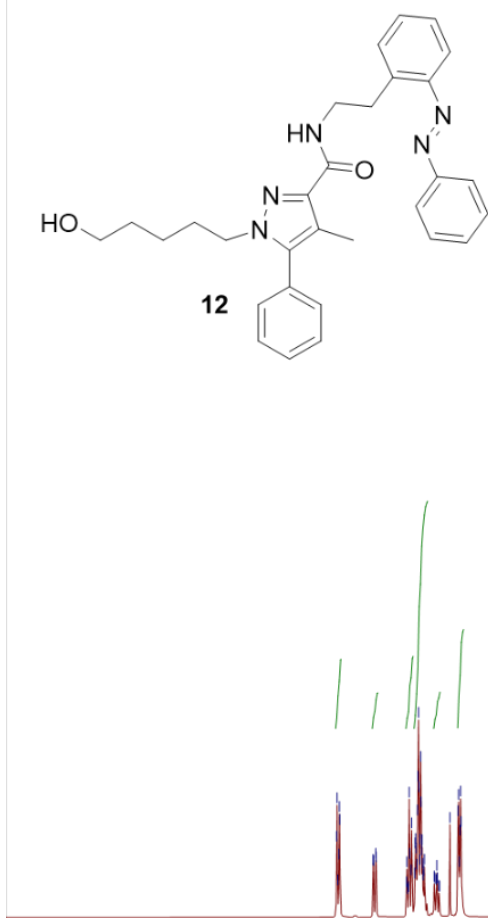

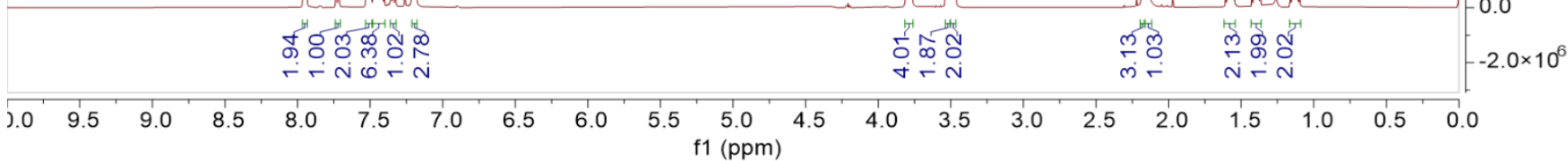

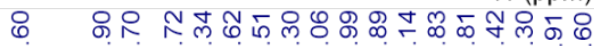

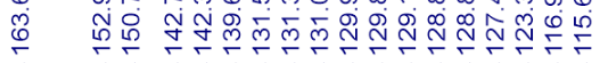

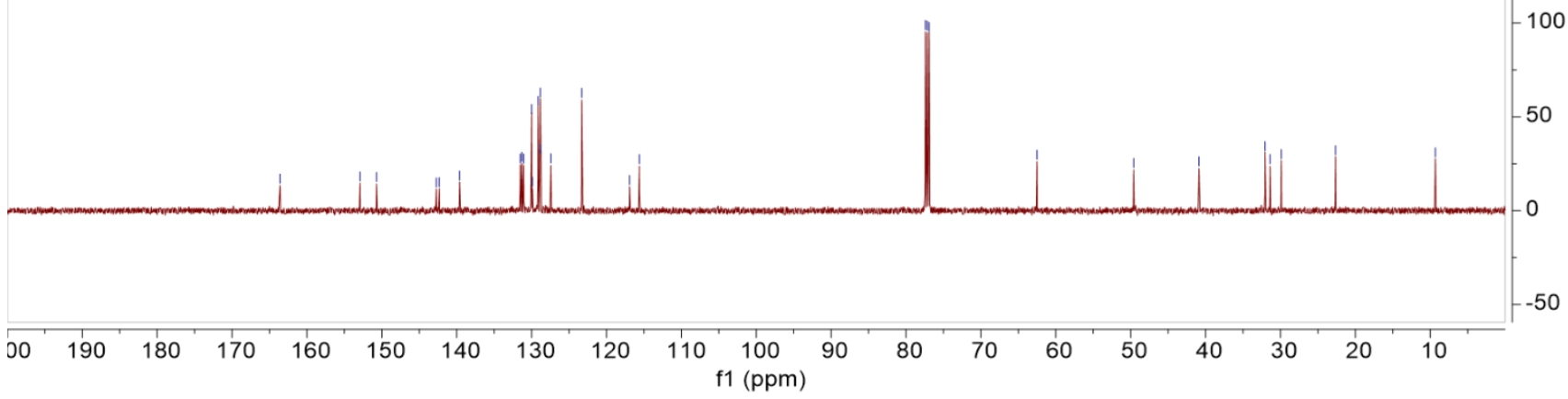



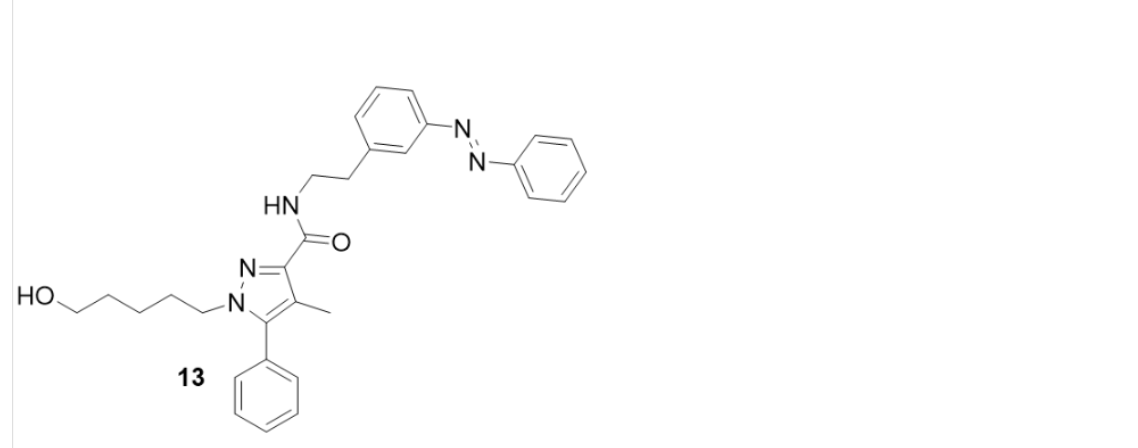

$3.0 \times 10^{7}$
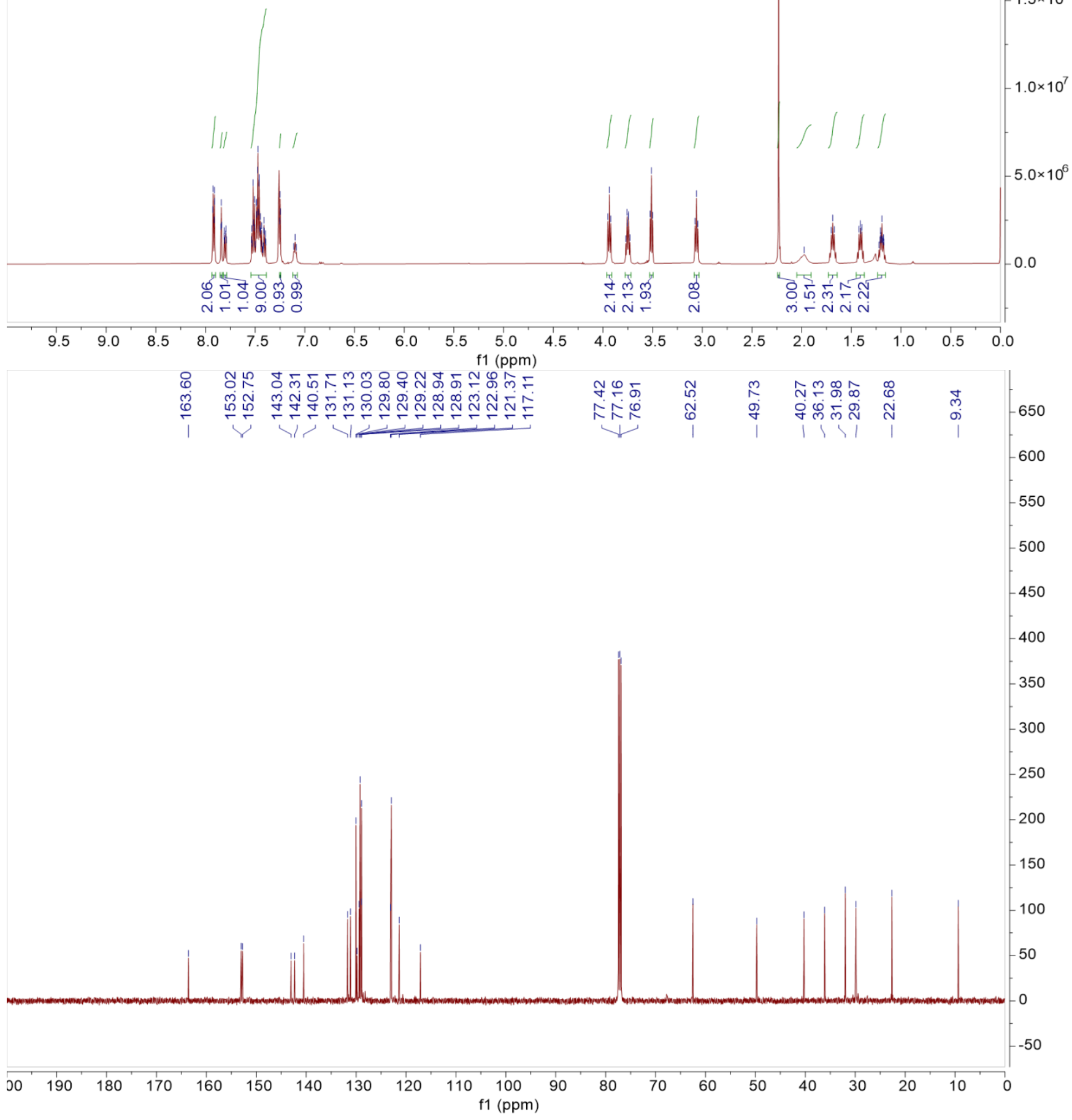

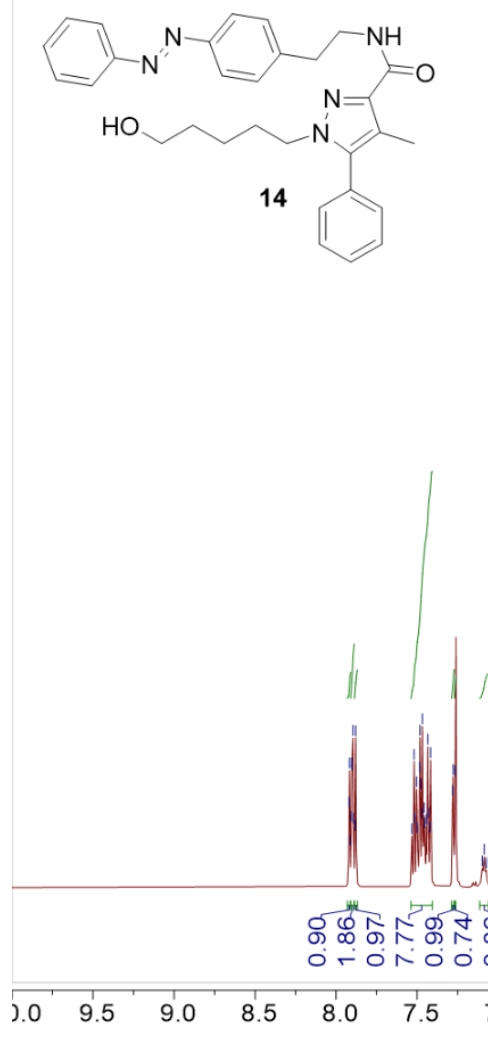

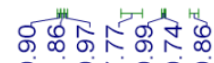

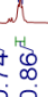

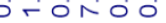

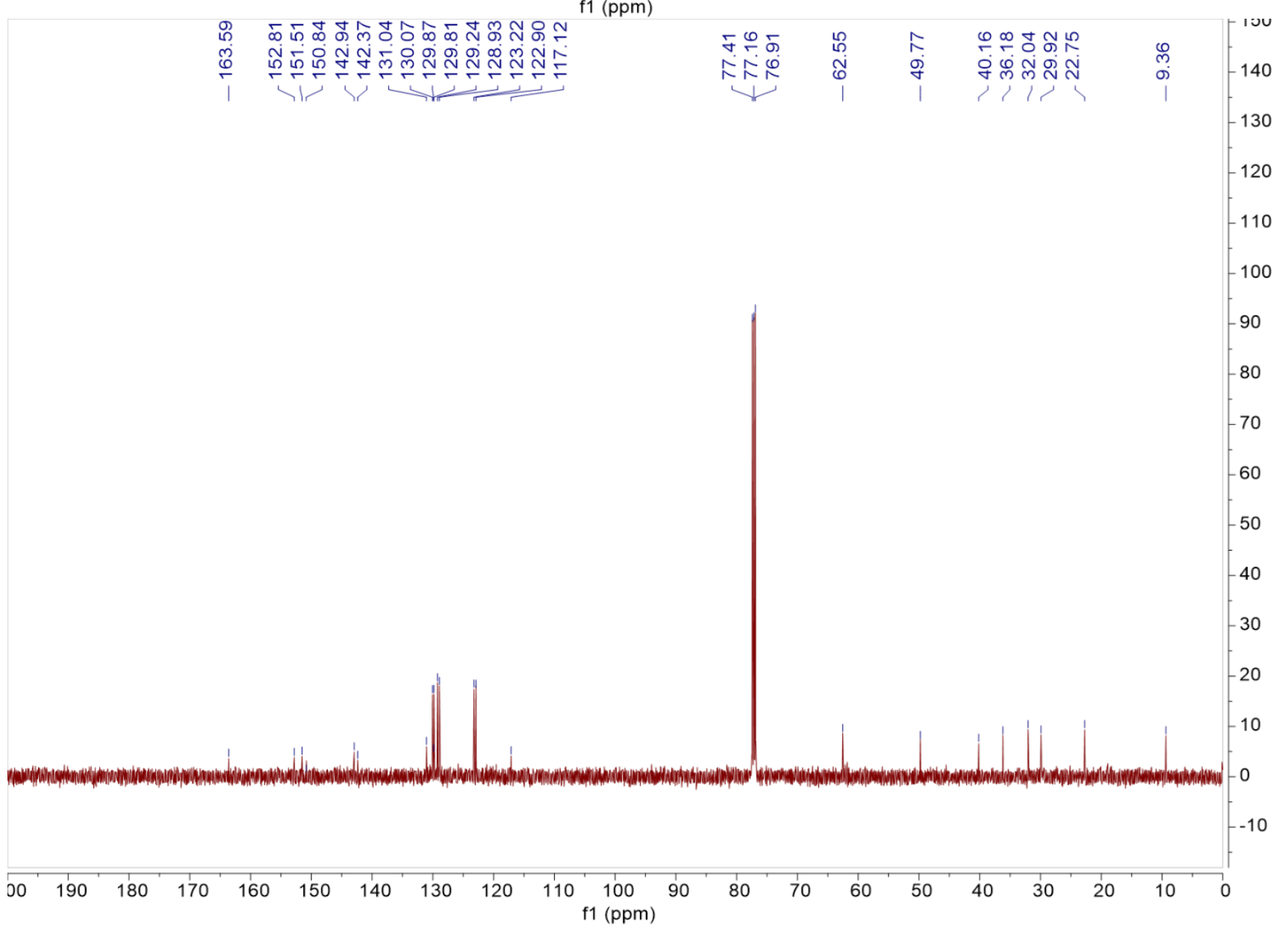




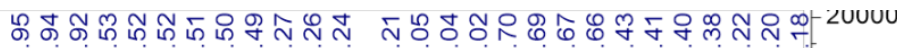

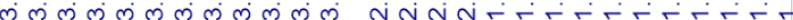
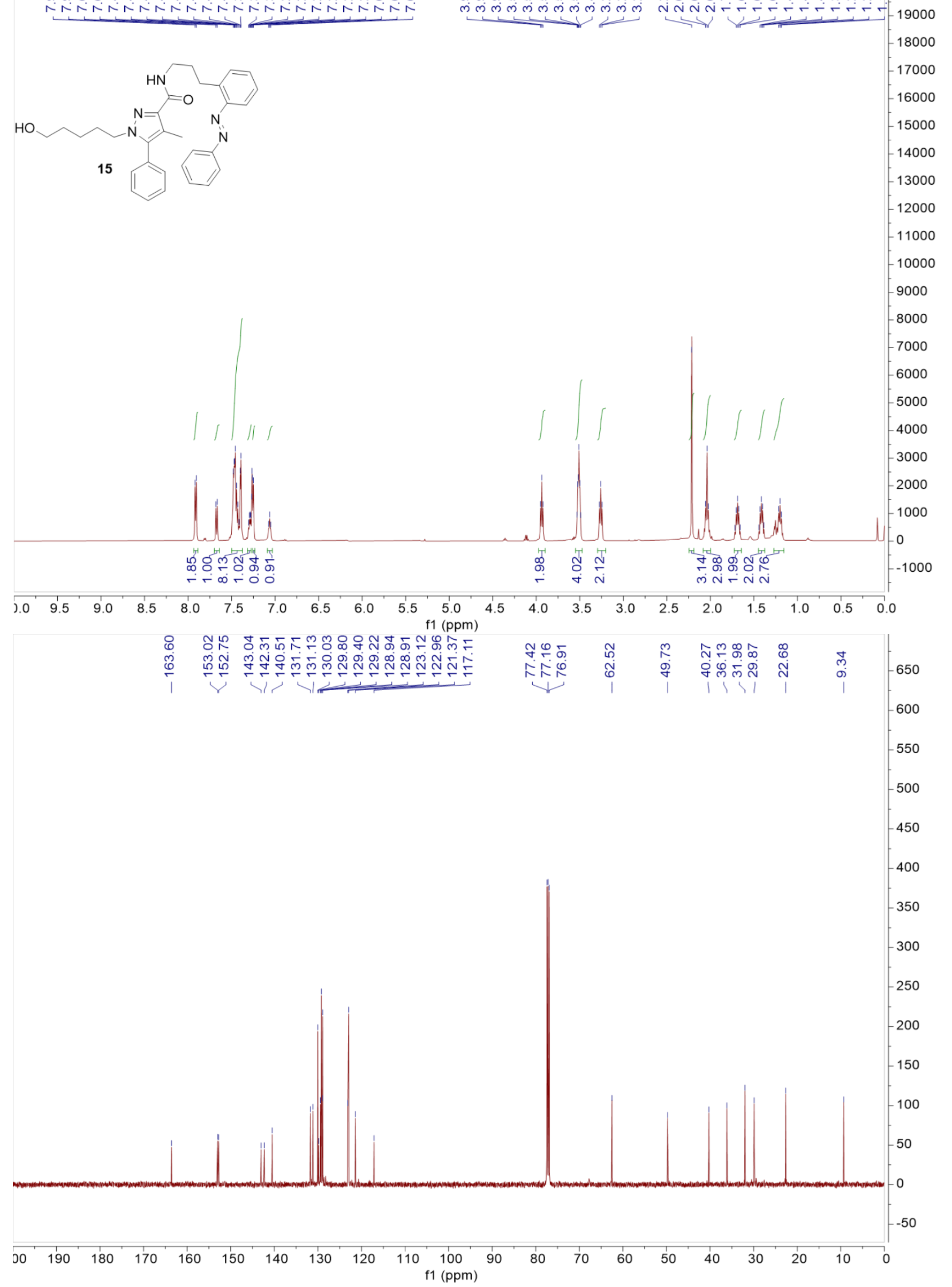

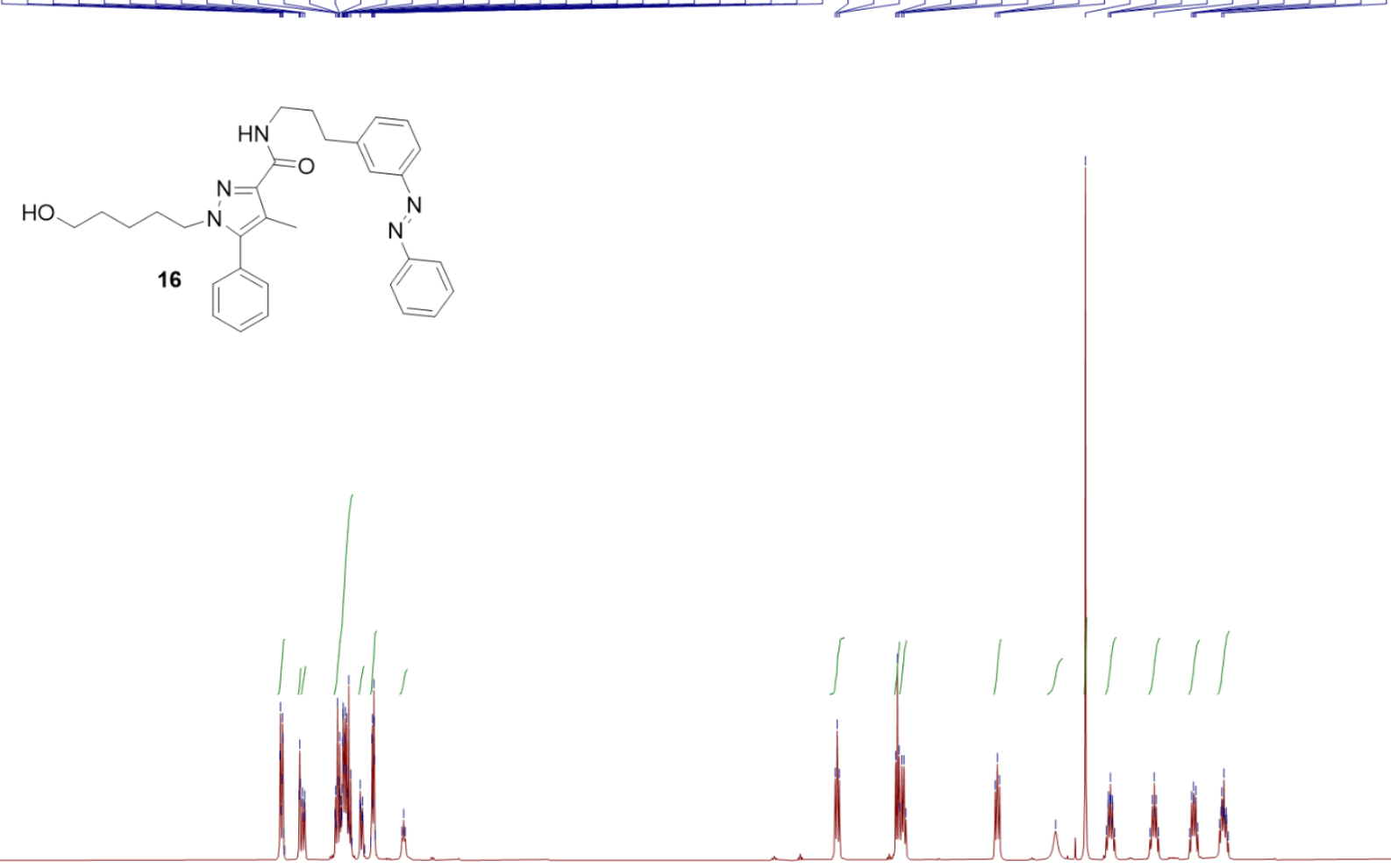

<ouvunur

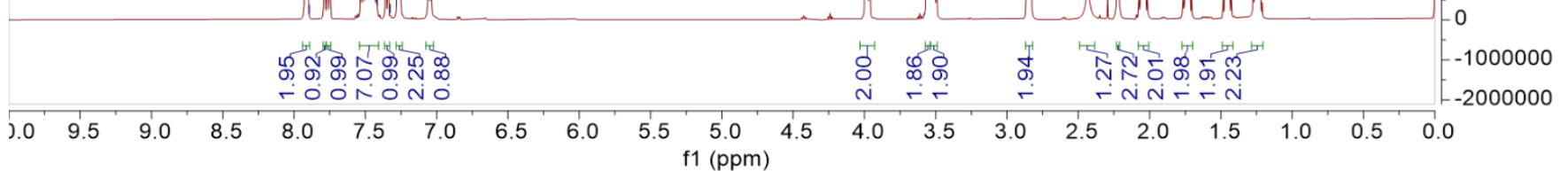

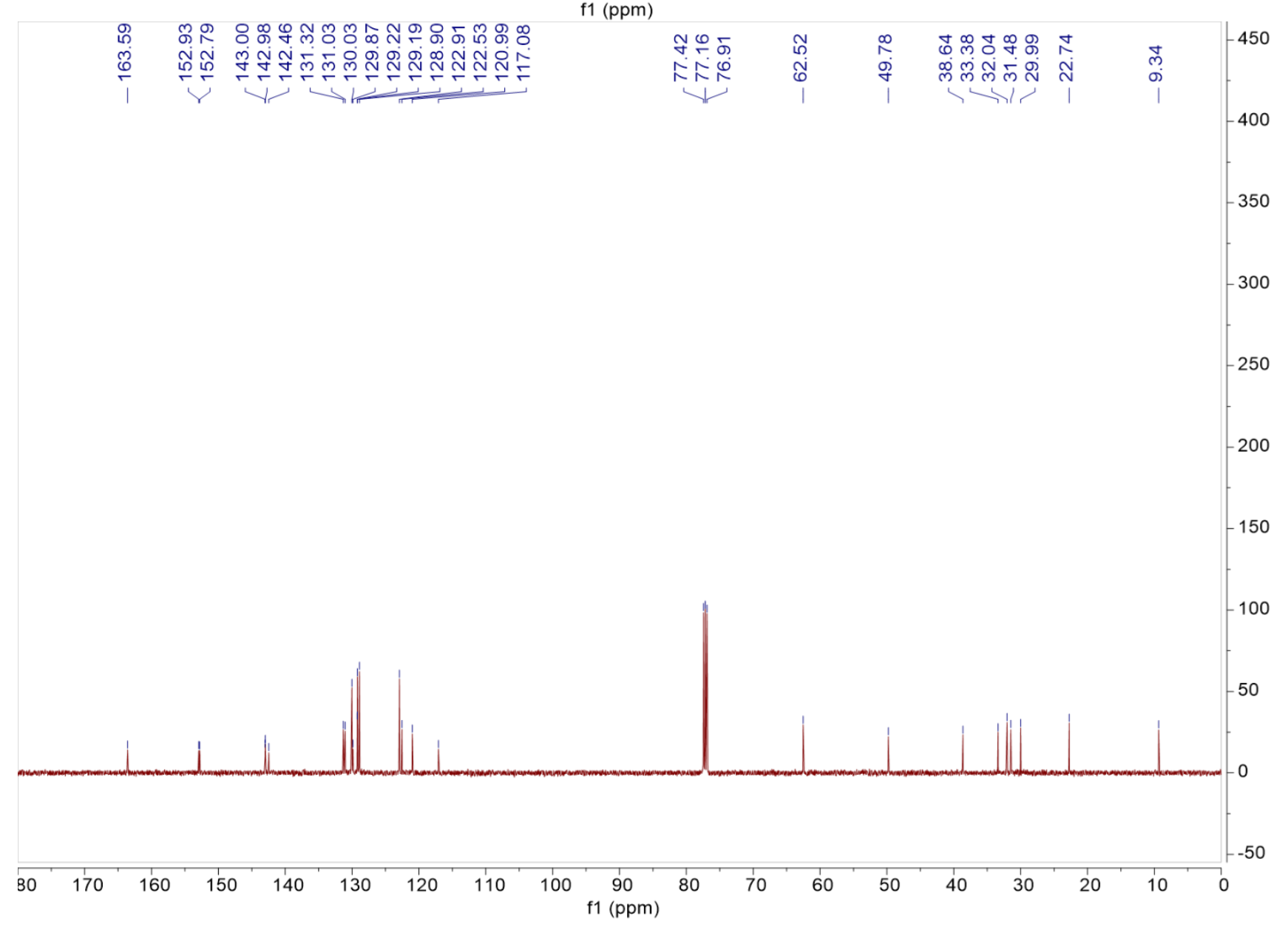



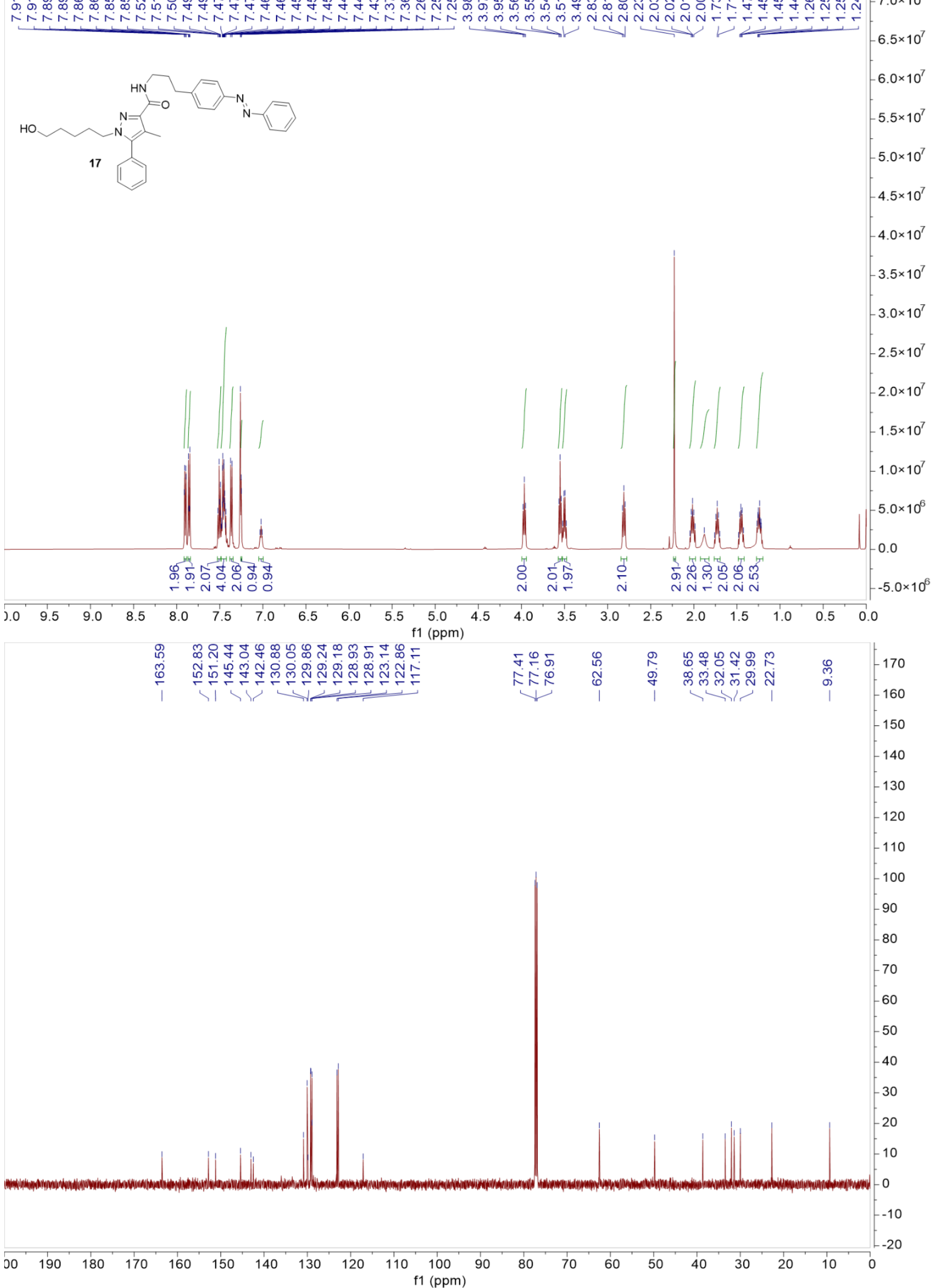


\section{Purification Characterization}

Method 1 was applied for AzoLig 6, 10-17; Method 2 was applied for AzoLig 7-8; Method 3 was applied for AzoLig 9. The detailed methods were described in general method.

\section{<PDA Ch1 254 nm>}

$\mathrm{mAU}$

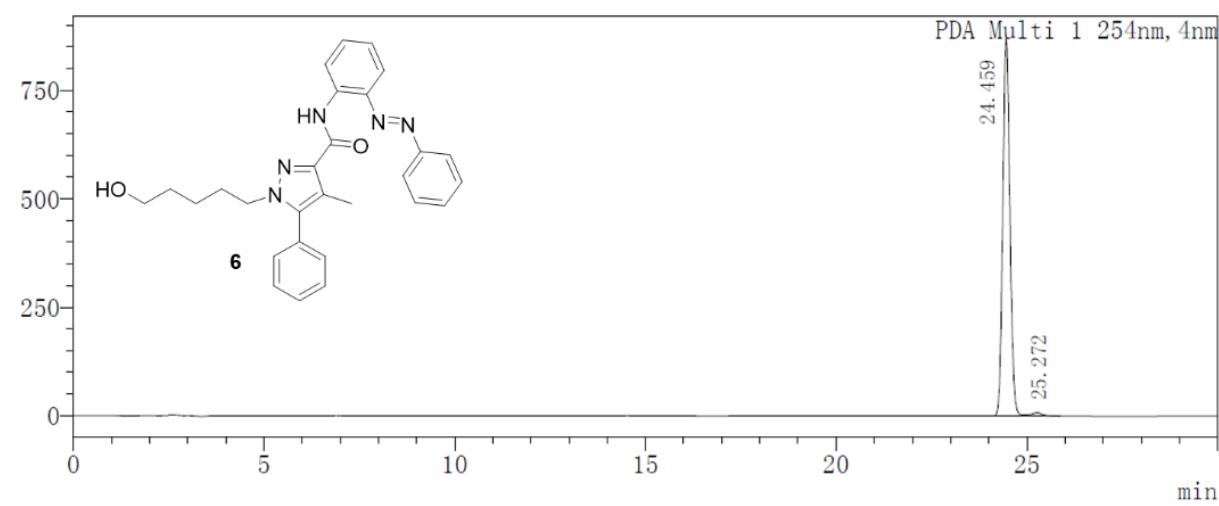

PDA Ch1 254nm

\begin{tabular}{|c|c|c|c|c|c|}
\hline Peak\# & Ret. Time (min) & Height(mAU) & Height $\%$ & Area $\left(\mathrm{mAU}^{*} \mathrm{~s}\right)$ & Area\% \\
\hline 1 & 24.459 & 872435 & 99.106 & 11274056 & 98.590 \\
\hline 2 & 25.272 & 7868 & 0.894 & 161199 & 1.410 \\
\hline Sum & & 880303 & 100.000 & 11435255 & 100.000 \\
\hline
\end{tabular}

\section{<PDA Ch1 254 nm>}

$\mathrm{mAU}$

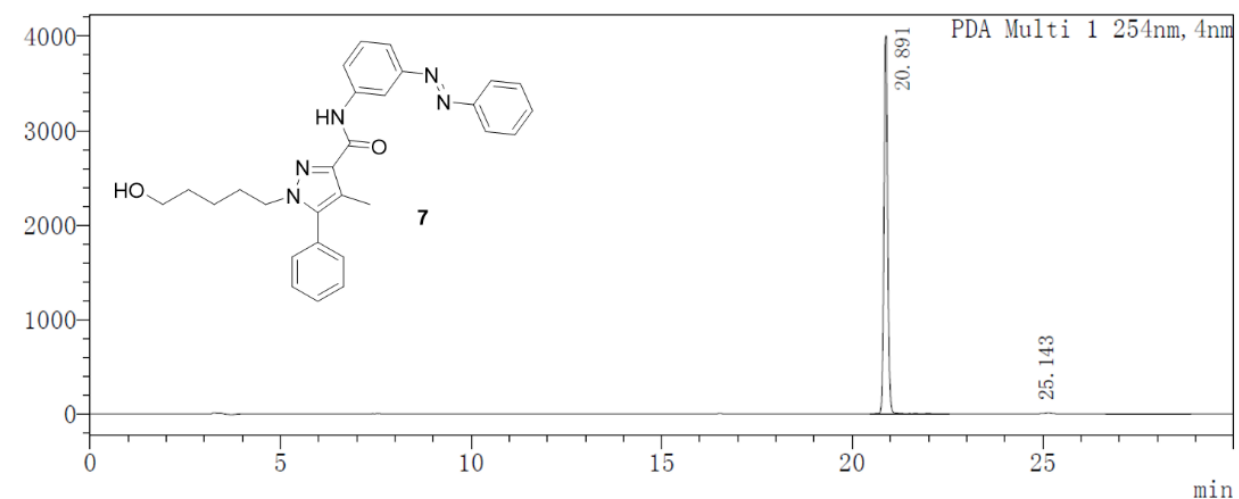

PDA Ch1 254nm

\begin{tabular}{|c|c|c|c|c|c|}
\hline Peak\# & Ret. Time (min) & Height(mAU) & Height $\%$ & Area $\left(\mathrm{mAU}^{*} \mathrm{~s}\right)$ & Area $\%$ \\
\hline 1 & 20.891 & 3999504 & 99.700 & 27240171 & 99.182 \\
\hline 2 & 25.143 & 12042 & 0.300 & 224554 & 0.818 \\
\hline Sum & & 4011546 & 100.000 & 27464725 & 100.000 \\
\hline
\end{tabular}


<PDA Ch1 254 nm>

mAU

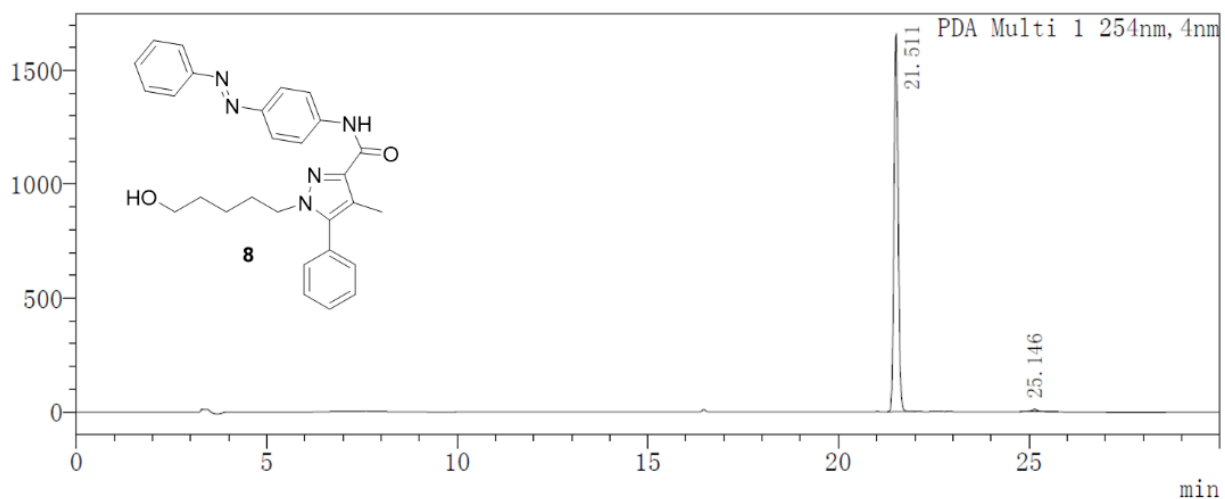

PDA Ch1 254nm

\begin{tabular}{|c|c|c|c|c|c|}
\hline Peak\# & Ret. Time (min) & Height(mAU) & Height $\%$ & Area(mAU*s) & Area\% \\
\hline 1 & 21.511 & 1653767 & 99.319 & 12584476 & 98.818 \\
\hline 2 & 25.146 & 11337 & 0.681 & 150519 & 1.182 \\
\hline Sum & & 1665105 & 100.000 & 12734995 & 100.000 \\
\hline
\end{tabular}

<PDA Ch1 254 nm>

$\mathrm{mAU}$

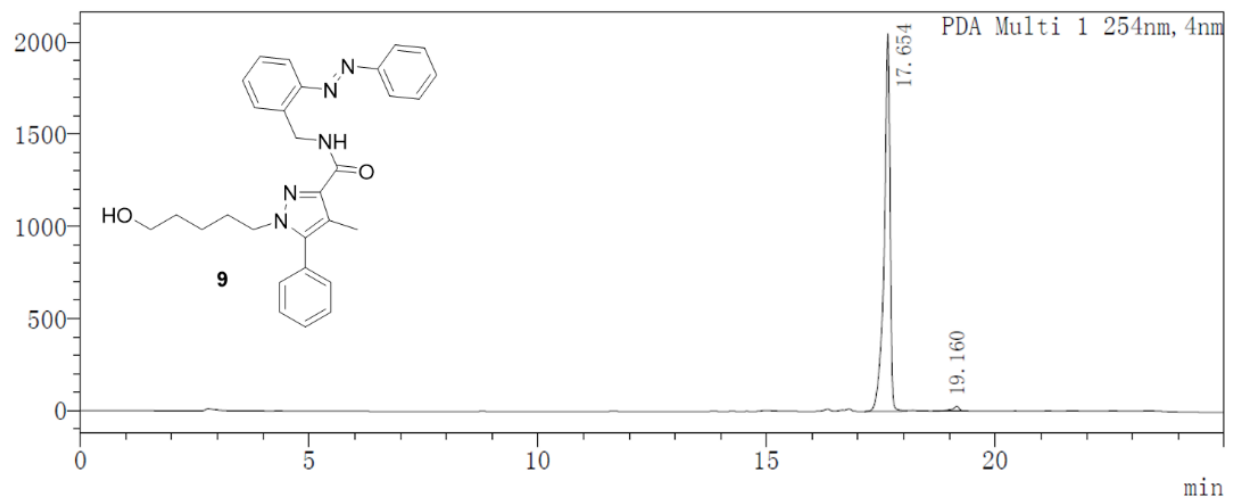

PDA Ch1 254nm

\begin{tabular}{|c|c|c|c|c|c|}
\hline Peak\# & Ret. Time $(\mathrm{min})$ & Height $(\mathrm{mAU})$ & Height $\%$ & Area(mAU*s) & Area\% \\
\hline 1 & 17.654 & 2050849 & 98.727 & 18109305 & 98.398 \\
\hline 2 & 19.160 & 26445 & 1.273 & 294923 & 1.602 \\
\hline Sum & & 2077293 & 100.000 & 18404228 & 100.000 \\
\hline
\end{tabular}


<PDA Ch1 254 nm>

$\mathrm{mAU}$

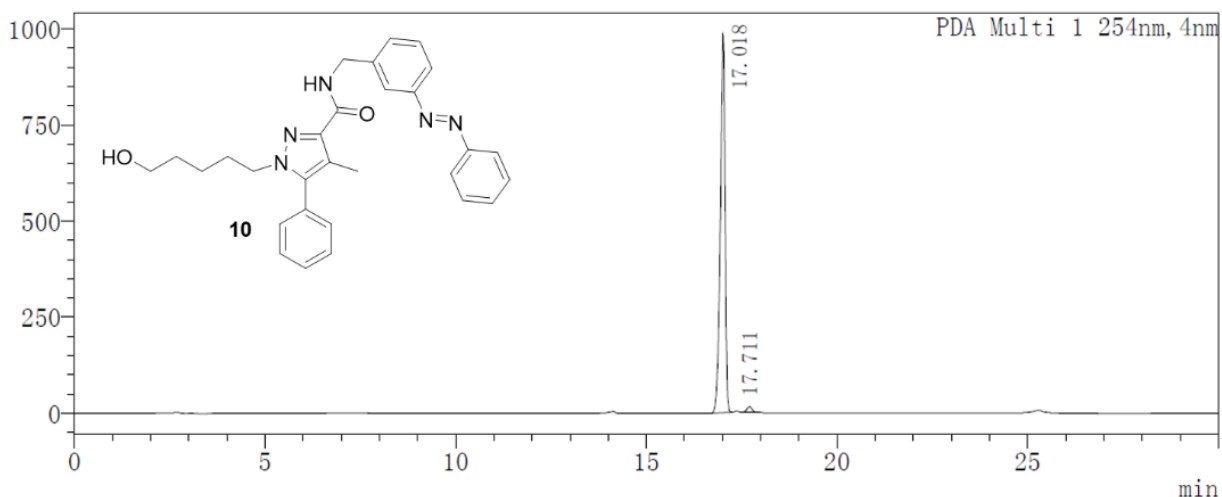

PDA Ch1 254nm

\begin{tabular}{|c|c|c|c|c|c|}
\hline Peak\# & Ret. Time (min) & Height(mAU) & Height $\%$ & Area $\left(\mathrm{mAU}^{*} \mathrm{~s}\right)$ & Area\% \\
\hline 1 & 17.018 & 984396 & 98.524 & 8468565 & 98.497 \\
\hline 2 & 17.711 & 14749 & 1.476 & 129245 & 1.503 \\
\hline Sum & & 999145 & 100.000 & 8597809 & 100.000 \\
\hline
\end{tabular}

<PDA Ch1 254 nm>

$\mathrm{mAU}$

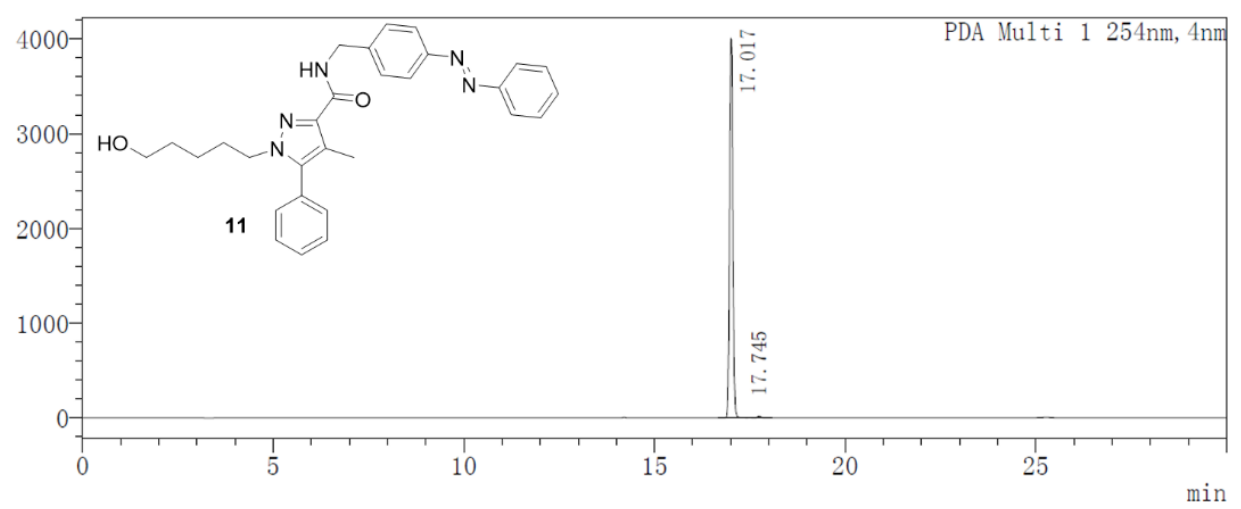

PDA Ch1 254nm

\begin{tabular}{|c|c|c|c|c|c|}
\hline Peak\# & Ret. Time (min) & Height(mAU) & Height $\%$ & Area $\left(\mathrm{mAU}^{*} \mathrm{~s}\right)$ & Area\% \\
\hline 1 & 17.017 & 3998534 & 99.593 & 23924793 & 99.582 \\
\hline 2 & 17.745 & 16351 & 0.407 & 100390 & 0.418 \\
\hline Sum & & 4014884 & 100.000 & 24025182 & 100.000 \\
\hline
\end{tabular}


<PDA Ch1 254 nm>

$\mathrm{mAU}$

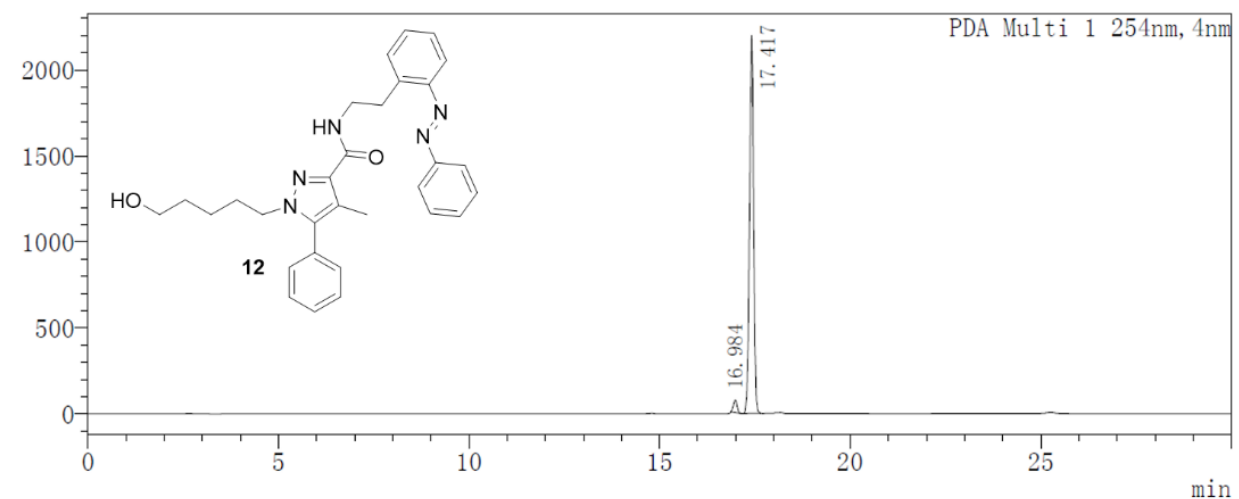

PDA Ch1 254nm

\begin{tabular}{|c|c|c|c|c|c|}
\hline Peak\# & Ret. Time (min) & Height(mAU) & Height $\%$ & Area(mAU*s) & Area\% \\
\hline 1 & 16.984 & 73098 & 3.216 & 483654 & 2.904 \\
\hline 2 & 17.417 & 2199540 & 96.784 & 16169764 & 97.096 \\
\hline Sum & & 2272638 & 100.000 & 16653418 & 100.000 \\
\hline
\end{tabular}

\section{<PDA Ch1 254 nm>}

$\mathrm{mAU}$

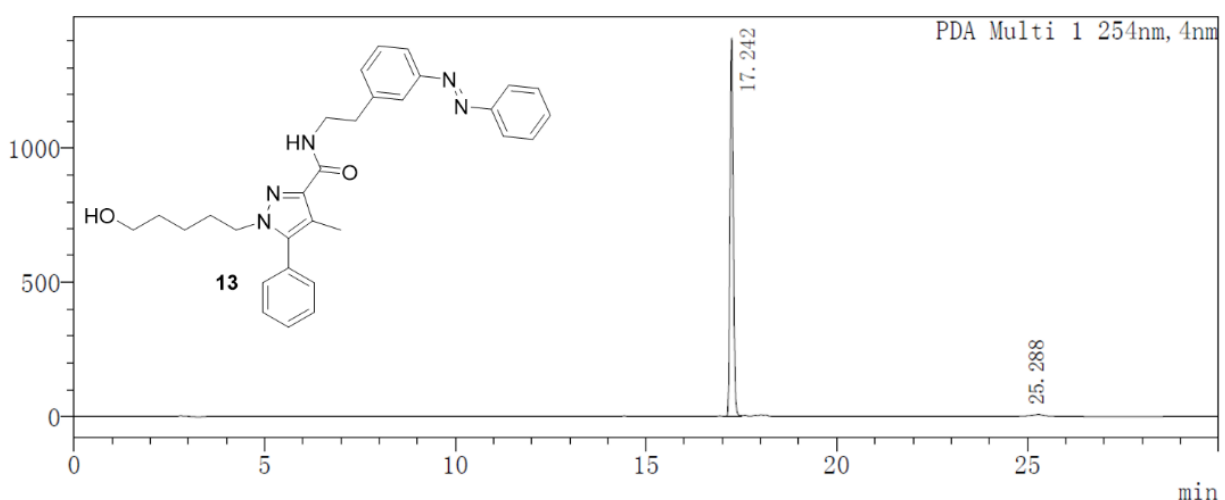

PDA Ch1 254nm

\begin{tabular}{|c|c|c|c|c|c|}
\hline Peak\# & Ret. Time (min) & Height(mAU) & Height $\%$ & Area(mAU*s) & Area\% \\
\hline 1 & 17.242 & 1409786 & 99.541 & 8327697 & 98.441 \\
\hline 2 & 25.288 & 6494 & 0.459 & 131920 & 1.559 \\
\hline Sum & & 1416280 & 100.000 & 8459617 & 100.000 \\
\hline
\end{tabular}


<PDA Ch1 254 nm>

$\mathrm{mAU}$

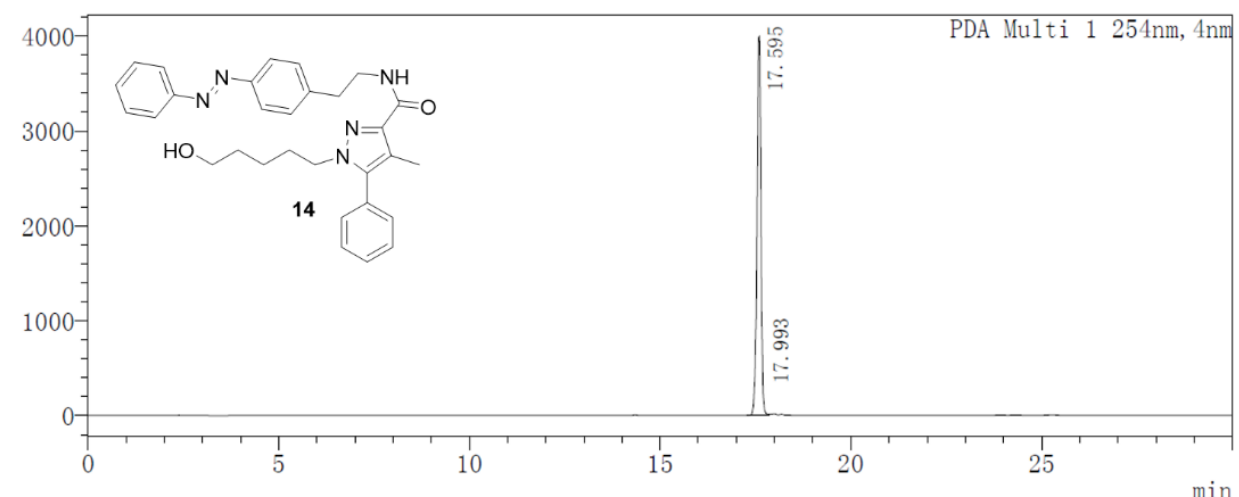

PDA Ch1 254nm

\begin{tabular}{|c|c|c|c|c|c|}
\hline Peak\# & Ret. Time (min) & Height(mAU) & Height $\%$ & Area $\left(\mathrm{mAU}{ }^{*} \mathrm{~s}\right)$ & Area\% \\
\hline 1 & 17.595 & 3998242 & 99.519 & 28722606 & 99.269 \\
\hline 2 & 17.993 & 19327 & 0.481 & 211602 & 0.731 \\
\hline Sum & & 4017570 & 100.000 & 28934208 & 100.000 \\
\hline
\end{tabular}

<PDA Ch1 254 nm>

$\mathrm{mAU}$

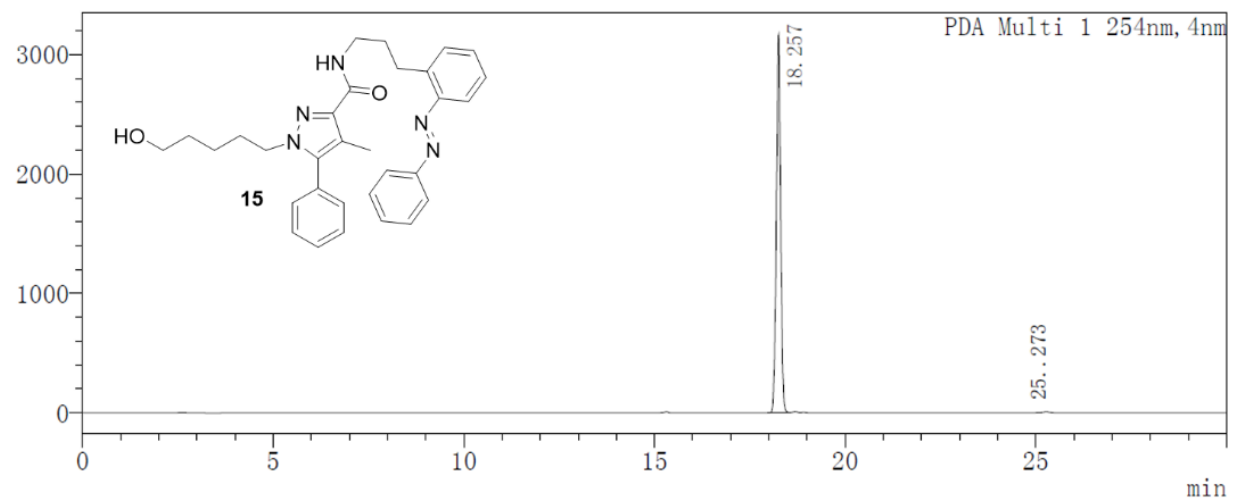

PDA Ch1 254nm

\begin{tabular}{|c|c|c|c|c|c|}
\hline Peak\# & Ret. Time (min) & Height $(\mathrm{mAU})$ & Height $\%$ & Area $\left(\mathrm{mAU}{ }^{*} \mathrm{~s}\right)$ & Area\% \\
\hline 1 & 18.257 & 3170992 & 99.727 & 24621895 & 99.494 \\
\hline 2 & 25.273 & 8696 & 0.273 & 125268 & 0.506 \\
\hline Sum & & 3179688 & 100.000 & 24747163 & 100.000 \\
\hline
\end{tabular}


<PDA Ch1 254 nm>

mAU

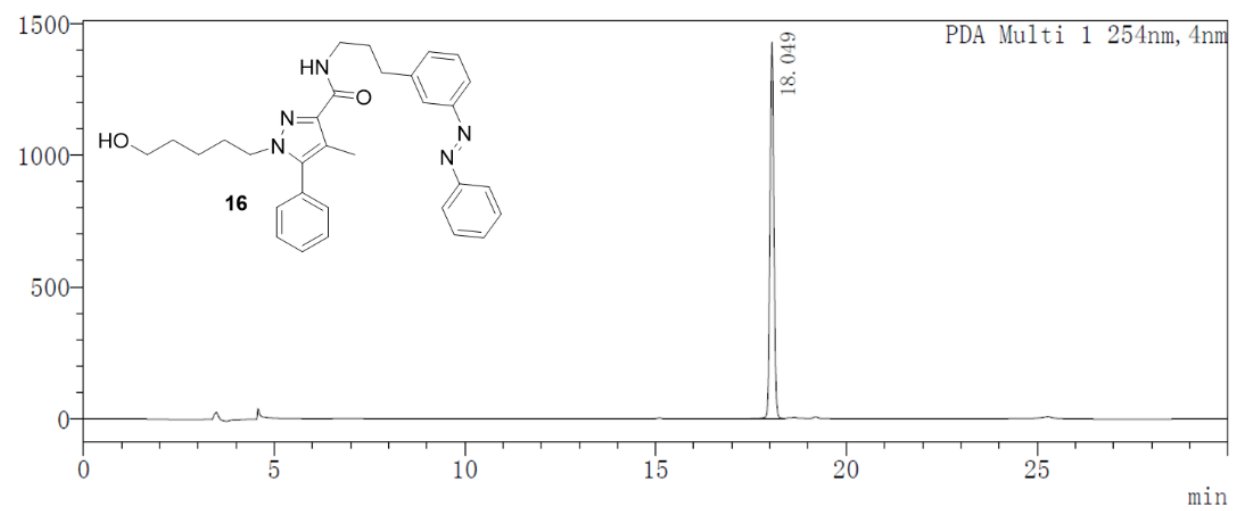

PDA Ch1 254nm

\begin{tabular}{|c|c|c|c|c|c|}
\hline Peak\# & Ret. Time (min) & Height(mAU) & Height\% & Area(mAU*s) & Area\% \\
\hline 1 & 18.049 & 1430340 & 100.000 & 10038296 & 100.000 \\
\hline Sum & & 1430340 & 100.000 & 10038296 & 100.000 \\
\hline
\end{tabular}

<PDA Ch1 254 nm>

$\mathrm{mAU}$

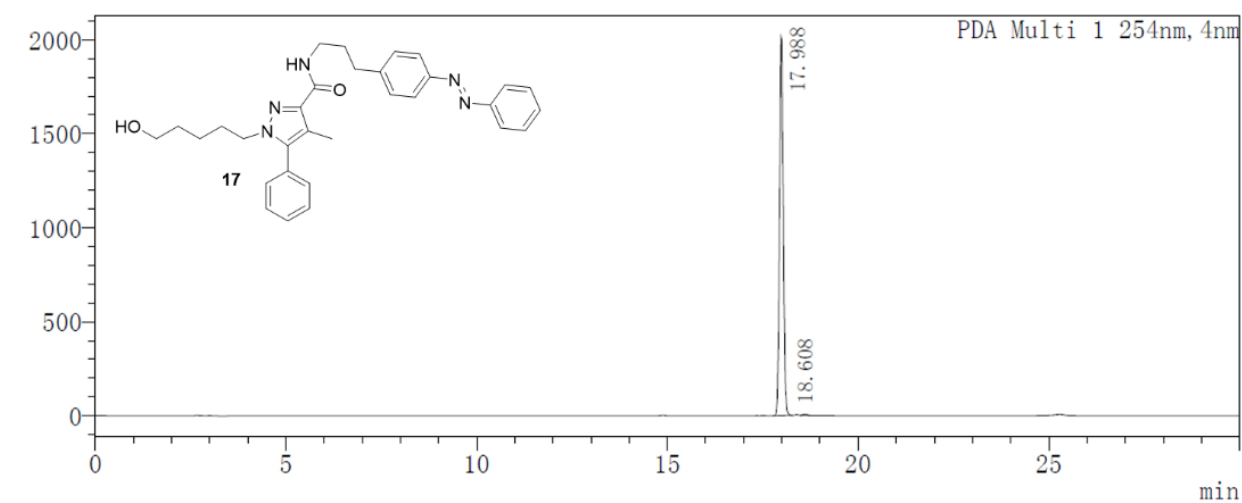

PDA Ch1 254nm

\begin{tabular}{|c|c|c|c|c|c|}
\hline Peak\# & Ret. Time (min) & Height(mAU) & Height $\%$ & Area $\left(\mathrm{mAU}^{*} \mathrm{~s}\right)$ & Area\% \\
\hline 1 & 17.988 & 2013436 & 99.690 & 13722983 & 99.682 \\
\hline 2 & 18.608 & 6261 & 0.310 & 43760 & 0.318 \\
\hline Sum & & 2019697 & 100.000 & 13766743 & 100.000 \\
\hline
\end{tabular}




\section{References}

(1) Huckvale, R.; Mortensen, M.; Pryde, D.; Smart, T. G.; Baker, J. R., Azogabazine: a Photochromic Antagonist of the GABAA Receptor. Org. Biomol. Chem. 2016, 14 (28), 6676-8.

(2) Borowiak, M.; Nahaboo, W.; Reynders, M.; Nekolla, K.; Jalinot, P.; Hasserodt, J.; Rehberg, M.; Delattre, M.; Zahler, S.; Vollmar, A.; Trauner, D.; Thorn-Seshold, O., Photoswitchable Inhibitors of Microtubule Dynamics Optically Control Mitosis and Cell Death. Cell 2015, $162(2), 403-411$.

(3) Broichhagen, J.; Johnston, N. R.; von Ohlen, Y.; Meyer-Berg, H.; Jones, B. J.; Bloom, S. R.; Rutter, G. A.; Trauner, D.; Hodson, D. J., Allosteric Optical Control of a Class B G-Protein-Coupled Receptor. Angew. Chem., Int. Ed. 2016, 55 (19), 5865-8; Angew. Chem. 2016, $128,5961-5965$.

(4) Cheng, B.; Morstein, J.; Ladefoged, L. K.; Maesen, J. B.; Schiøtt, B.; Sinning, S.; Trauner, D., A Photoswitchable Inhibitor of the Human Serotonin Transporter. ACS Chem. Neurosci. 2020, 11 (9), 1231-1237.

(5) Cheng, B.; Shchepakin, D.; Kavanaugh, M. P.; Trauner, D., Photoswitchable Inhibitor of a Glutamate Transporter. ACS Chem. Neurosci. 2017, 8 (8), 1668-1672.

(6) Arkhipova, V.; Fu, H.; Hoorens, M. W. H.; Trinco, G.; Lameijer, L. N.; Marin, E.; Feringa, B. L.; Poelarends, G. J.; Szymanski, W.; Slotboom, D. J.; Guskov, A., Structural Aspects of Photopharmacology: Insight into the Binding of Photoswitchable and Photocaged Inhibitors to the Glutamate Transporter Homologue. J. Am. Chem. Soc. 2021, 143 (3), 1513-1520.

(7) Damijonaitis, A.; Broichhagen, J.; Urushima, T.; Hull, K.; Nagpal, J.; Laprell, L.; Schonberger, M.; Woodmansee, D. H.; Rafiq, A.; Sumser, M. P.; Kummer, W.; Gottschalk, A.; Trauner, D., AzoCholine Enables Optical Control of Alpha 7 Nicotinic Acetylcholine Receptors in Neural Networks. ACS Chem. Neurosci. 2015, 6 (5), 701-7.

(8) Eisel, B.; Hartrampf, F. W. W.; Meier, T.; Trauner, D., Reversible Optical Control of F1 Fo -ATP Synthase Using Photoswitchable Inhibitors. FEBS Lett. 2018, 592 (3), 343-355.

(9) Ferreira, R.; Nilsson, J. R.; Solano, C.; Andréasson, J.; Grøtli, M., Design, Synthesis and Inhibitory Activity of Photoswitchable RET Kinase Inhibitors. Sci. Rep. 2015, 5, 9769-9776.

(10) Frank, J. A.; Yushchenko, D. A.; Fine, N. H. F.; Duca, M.; Citir, M.; Broichhagen, J.; Hodson, D. J.; Schultz, C.; Trauner, D., Optical Control of GPR40 Signalling in Pancreatic Beta-cells. Chem. Sci. 2017, 8 (11), 7604-7610.

(11) Gomez-Santacana, X.; Pittolo, S.; Rovira, X.; Lopez, M.; Zussy, C.; Dalton, J. A.; Faucherre, A.; Jopling, C.; Pin, J. P.; Ciruela, F.; Goudet, C.; Giraldo, J.; Gorostiza, P.; Llebaria, A., Illuminating Phenylazopyridines to Photoswitch Metabotropic Glutamate Receptors: From the Flask to the Animals. ACS Cent. Sci. 2017, 3 (1), 81-91.

(12) Pittolo, S.; Gomez-Santacana, X.; Eckelt, K.; Rovira, X.; Dalton, J.; Goudet, C.; Pin, J. P.; Llobet, A.; Giraldo, J.; Llebaria, A.; Gorostiza, P., An Allosteric Modulator to Control Endogenous G protein-coupled Receptors with Light. Nat. Chem. Biol. 2014, 10 (10), 813-5.

(13) Grathwol, C. W.; Wossner, N.; Behnisch-Cornwell, S.; Schulig, L.; Zhang, L.; Einsle, O.; Jung, M.; Link, A., Activation of Sirtuin 2 Inhibitors Employing Photoswitchable Geometry and Aqueous Solubility. ChemMedChem. 2020, 15 (15), 1480-1489.

(14) Hauwert, N. J.; Mocking, T. A. M.; Da Costa Pereira, D.; Lion, K.; Huppelschoten, Y.; Vischer, H. F.; De Esch, I. J. P.; Wijtmans, M.; Leurs, R., A Photoswitchable Agonist for the Histamine H3 Receptor, a Prototypic Family A G-Protein-Coupled Receptor. Angew. Chem., Int. Ed. 2019, 58 (14), 4531-4535; Angew. Chem. 2019, 131, 4579-4583.

(15) Hoorens, M. W. H.; Ourailidou, M. E.; Rodat, T.; van der Wouden, P. E.; Kobauri, P.; Kriegs, M.; Peifer, C.; Feringa, B. L.; Dekker, F. J.; Szymanski, W., Light-controlled Inhibition of BRAFV600E Kinase. Eur. J. Med. Chem. 2019, 179, 133-146.

(16) Matera, C.; Gomila, A. M. J.; Camarero, N.; Libergoli, M.; Soler, C.; Gorostiza, P., Photoswitchable Antimetabolite for Targeted Photoactivated Chemotherapy. J. Am. Chem. Soc. 2018, 140 (46), 15764-15773.

(17) Schoenberger, M.; Damijonaitis, A.; Zhang, Z.; Nagel, D.; Trauner, D., Development of a New Photochromic Ion Channel Blocker via Azologization of Fomocaine. ACS Chem. Neurosci. 2014, 5 (7), 514-8.

(18) Morstein, J.; Awale, M.; Reymond, J. L.; Trauner, D., Mapping the Azolog Space Enables the Optical Control of New Biological Targets. ACS Cent Sci 2019, 5 (4), 607-618.

(19) Morstein, J.; Trads, J. B.; Hinnah, K.; Willems, S.; Barber, D. M.; Trauner, M.; Merk, D.; Trauner, D., Optical Control of the Nuclear Bile Acid Receptor FXR with a Photohormone. Chem. Sci. 2020, 11 (2), 429-434.

(20) Tsuchiya, K.; Umeno, T.; Tsuji, G.; Yokoo, H.; Tanaka, M.; Fukuhara, K.; Demizu, Y.; Misawa, T., Development of Photoswitchable Estrogen Receptor Ligands. Chem. Pharm. Bull. (Tokyo) 2020, 68 (4), 398-402.

(21) Mostyn, S. N.; Sarker, S.; Muthuraman, P.; Raja, A.; Shimmon, S.; Rawling, T.; Cioffi, C. L.; Vandenberg, R. J., Photoswitchable ORG25543 Congener Enables Optical Control of Glycine Transporter 2. ACS Chem. Neurosci. 2020, 11 (9), 1250-1258.

(22) Weston, C. E.; Kramer, A.; Colin, F.; Yildiz, O.; Baud, M. G.; Meyer-Almes, F. J.; Fuchter, M. J., Toward Photopharmacological Antimicrobial Chemotherapy Using Photoswitchable Amidohydrolase Inhibitors. ACS Infect. Dis. 2017, 3 (2), 152-161.

(23) Westphal, M. V.; Schafroth, M. A.; Sarott, R. C.; Imhof, M. A.; Bold, C. P.; Leippe, P.; Dhopeshwarkar, A.; Grandner, J. M.; Katritch, V.; Mackie, K.; Trauner, D.; Carreira, E. M.; Frank, J. A., Synthesis of Photoswitchable Delta(9)-Tetrahydrocannabinol Derivatives Enables Optical Control of Cannabinoid Receptor 1 Signaling. J. Am. Chem. Soc. 2017, 139 (50), 18206-18212.

(24) Hauwert, N. J.; Mocking, T. A. M.; Da Costa Pereira, D.; Kooistra, A. J.; Wijnen, L. M.; Vreeker, G. C. M.; Verweij, E. W. E.; De Boer, A. H.; Smit, M. J.; De Graaf, C.; Vischer, H. F.; de Esch, I. J. P.; Wijtmans, M.; Leurs, R., Synthesis and Characterization of a Bidirectional Photoswitchable Antagonist Toolbox for Real-Time GPCR Photopharmacology. J. Am. Chem. Soc. 2018, 140 (12), $4232-4243$. (25) Blanco, B.; Palasis, K. A.; Adwal, A.; Callen, D. F.; Abell, A. D., Azobenzene-containing Photoswitchable Proteasome Inhibitors with Selective Activity and Cellular Toxicity. Bioorg. Med. Chem. 2017, 25 (19), 5050-5054.

(26) Gómez-Santacana, X.; de Munnik, S. M.; Vijayachandran, P.; Da Costa Pereira, D.; Bebelman, J. P. M.; de Esch, I. J. P.; Vischer, H. F.; Wijtmans, M.; Leurs, R., Photoswitching the Efficacy of a Small-Molecule Ligand for a Peptidergic GPCR: from Antagonism to Agonism. Angew. Chem., Int. Ed. 2018, 57 (36), 11608-11612; Angew. Chem. 2018, 130, 11782-11786.

(27) Gomez-Santacana, X.; de Munnik, S. M.; Mocking, T. A. M.; Hauwert, N. J.; Sun, S.; Vijayachandran, P.; de Esch, I. J. P.; Vischer, H. F.; Wijtmans, M.; Leurs, R., A Toolbox of Molecular Photoswitches to Modulate the CXCR3 Chemokine Receptor with Light. Beilstein J. Org. Chem. 2019, 15, 2509-2523. 
(28) Lutz, T.; Wein, T.; Hofner, G.; Pabel, J.; Eder, M.; Dine, J.; Wanner, K. T., Development of New Photoswitchable Azobenzene Based Gamma-Aminobutyric Acid (GABA) Uptake Inhibitors with Distinctly Enhanced Potency upon Photoactivation. J. Med. Chem. 2018, 61 (14), 6211-6235.

(29) Yeoh, Y. Q.; Horsley, J. R.; Yu, J.; Polyak, S. W.; Jovcevski, B.; Abell, A. D., Short Photoswitchable Antibacterial Peptides. ChemMedChem 2020, 15, 1505-1508.

(30) Duran-Corbera, A.; Catena, J.; Otero-Viñas, M.; Llebaria, A.; Rovira, X., Photoswitchable Antagonists for a Precise Spatiotemporal Control of $\beta 2$-Adrenoceptors. J. Med. Chem. 2020, 63 (15), 8458-8470.

(31) Hartrampf, F. W. W.; Barber, D. M.; Gottschling, K.; Leippe, P.; Hollmann, M.; Trauner, D., Development of a Photoswitchable Antagonist of NMDA Receptors. Tetrahedron 2017, 73 (33), 4905-4912.

(32) Quandt, G.; Hofner, G.; Pabel, J.; Dine, J.; Eder, M.; Wanner, K. T., First Photoswitchable Neurotransmitter Transporter Inhibitor: Light-induced Control of Gamma-aminobutyric Acid Transporter 1 (GAT1) Activity in Mouse Brain. J. Med. Chem. 2014, 57 (15), 68096821.

(33) Kolarski, D.; Miller, S.; Oshima, T.; Nagai, Y.; Aoki, Y.; Kobauri, P.; Srivastava, A.; Sugiyama, A.; Amaike, K.; Sato, A.; Tama, F.; Szymanski, W.; Feringa, B. L.; Itami, K.; Hirota, T., Photopharmacological Manipulation of Mammalian CRY1 for Regulation of the Circadian Clock. J. Am. Chem. Soc. 2021, 143 (4), 2078-2087.

(34) Frank, J. A.; Moroni, M.; Moshourab, R.; Sumser, M.; Lewin, G. R.; Trauner, D., Photoswitchable Fatty Acids Enable Optical Control of TRPV1. Nat. Commun. 2015, 6, 7118-7128.

(35) Frank, J. A.; Yushchenko, D. A.; Hodson, D. J.; Lipstein, N.; Nagpal, J.; Rutter, G. A.; Rhee, J. S.; Gottschalk, A.; Brose, N.; Schultz, C.; Trauner, D., Photoswitchable Diacylglycerols Enable Optical Control of Protein Kinase C. Nat. Chem. Biol. 2016, 12 (9), 755-62.

(36) Hartrampf, N.; Seki, T.; Baumann, A.; Watson, P.; Veprek, N. A.; Hetzler, B. E.; Hoffmann-Roder, A.; Tsuji, M.; Trauner, D., Optical Control of Cytokine Production Using Photoswitchable Galactosylceramides. Chemistry 2020, 26 (20), 4476-4479.

(37) Kol, M.; Williams, B.; Toombs-Ruane, H.; Franquelim, H. G.; Korneev, S.; Schroeer, C.; Schwille, P.; Trauner, D.; Holthuis, J. C.; Frank, J. A., Optical Manipulation of Sphingolipid Biosynthesis Using Photoswitchable Ceramides. eLife 2019, 8, 1-30.

(38) Morstein, J.; Dacheux, M. A.; Norman, D. D.; Shemet, A.; Donthamsetti, P. C.; Citir, M.; Frank, J. A.; Schultz, C.; Isacoff, E. Y.; Parrill, A. L.; Tigyi, G. J.; Trauner, D., Optical Control of Lysophosphatidic Acid Signaling. J. Am. Chem. Soc. 2020, 142 (24), 1061210616.

(39) Morstein, J.; Hill, R. Z.; Novak, A. J. E.; Feng, S.; Norman, D. D.; Donthamsetti, P. C.; Frank, J. A.; Harayama, T.; Williams, B. M.; Parrill, A. L.; Tigyi, G. J.; Riezman, H.; Isacoff, E. Y.; Bautista, D. M.; Trauner, D., Optical Control of Sphingosine-1-phosphate Formation and Function. Nat. Chem. Biol. 2019, 15 (6), 623-631.

(40) Tiapko, O.; Shrestha, N.; Lindinger, S.; Guedes de la Cruz, G.; Graziani, A.; Klec, C.; Butorac, C.; Graier, W. F.; Kubista, H.; Freichel, M.; Birnbaumer, L.; Romanin, C.; Glasnov, T.; Groschner, K., Lipid-independent Control of Endothelial and Neuronal TRPC3 Channels by Light. Chem. Sci. 2019, 10 (9), 2837-2842.

(41) Sarott, R. C.; Viray, A. E. G.; Pfaff, P.; Sadybekov, A.; Rajic, G.; Katritch, V.; Carreira, E. M.; Frank, J. A., Optical Control of Cannabinoid Receptor 2-Mediated Ca ${ }^{2+}$ Release Enabled by Synthesis of Photoswitchable Probes. J. Am. Chem. Soc. 2021, 143, $736-743$.

(42) Rodríguez-Soacha, D. A.; Fender, J.; Ramírez, Y. A.; Collado, J. A.; Muñoz, E.; Maitra, R.; Sotriffer, C.; Lorenz, K.; Decker, M., "Photo-Rimonabant": Synthesis and Biological Evaluation of Novel Photoswitchable Molecules Derived from Rimonabant Lead to a Highly Selective and Nanomolar "Cis-On" CB1R Antagonist. ACS Chem. Neurosci. 2021, 12 (9), 1632-1647.

(43) Willems, S.; Morstein, J.; Hinnah, K.; Trauner, D.; Merk, D., A Photohormone for Light-Dependent Control of PPAR $\alpha$ in Live Cells. J. Med. Chem. 2021.

(44) Riefolo, F.; Sortino, R.; Matera, C.; Claro, E.; Preda, B.; Vitiello, S.; Traserra, S.; Jiménez, M.; Gorostiza, P., Rational Design of Photochromic Analogues of Tricyclic Drugs. J. Med. Chem. 2021, 64 (13), 9259-9270.

(45) Donthamsetti, P.; Konrad, D. B.; Hetzler, B.; Fu, Z.; Trauner, D.; Isacoff, E. Y., Selective Photoswitchable Allosteric Agonist of a G Protein-Coupled Receptor. J. Am. Chem. Soc. 2021, 143 (24), 8951-8956.

(46) Kobauri, P.; Szymanski, W.; Cao, F.; Thallmair, S.; Marrink, S. J.; Witte, M. D.; Dekker, F. J.; Feringa, B. L., Biaryl Sulfonamides as Cisoid Azosteres for Photopharmacology. Chem. Commun. 2021, 57 (34), 4126-4129.

(47) Dolles, D.; Strasser, A.; Wittmann, H.-J.; Marinelli, O.; Nabissi, M.; Pertwee, R. G.; Decker, M., The First Photochromic Affinity Switch for the Human Cannabinoid Receptor 2. Adv. Ther. 2018, 1 (1), 1-6.

(48) Prischich, D.; Gomila, A. M. J.; Milla-Navarro, S.; Sangüesa, G.; Diez-Alarcia, R.; Preda, B.; Matera, C.; Batlle, M.; Ramírez, L.; Giralt, E.; Hernando, J.; Guasch, E.; Meana, J. J.; de la Villa, P.; Gorostiza, P., Adrenergic Modulation With Photochromic Ligands. Angew. Chem. Int. Ed. 2021, 60 (7), 3625-3631.

(49) Kolarski, D.; Sugiyama, A.; Rodat, T.; Schulte, A.; Peifer, C.; Itami, K.; Hirota, T.; Feringa, B. L.; Szymanski, W., Reductive Stability Evaluation of 6-azopurine Photoswitches for the Regulation of CKI $\alpha$ Activity and Circadian Rhythms. Org. Biomol. Chem. 2021, 19 (10), 2312-2321.

(50) Rustler, K.; Maleeva, G.; Bregestovski, P.; König, B., Azologization of Serotonin 5-HT3 Receptor Antagonists. Beilstein J. Org. Chem. 2019, 15, 780-788.

(51) Barber, D. M.; Liu, S.-A.; Gottschling, K.; Sumser, M.; Hollmann, M.; Trauner, D., Optical Control of AMPA Receptors Using a Photoswitchable Quinoxaline-2,3-dione Antagonist. Chem. Sci. 2017, 8 (1), 611-615.

(52) Berman, H. M.; Westbrook, J.; Feng, Z.; Gilliland, G.; Bhat, T. N.; Weissig, H.; Shindyalov, I. N.; Bourne, P. E., The Protein Data Bank. Nucleic Acids Res. 2000, 28 (1), 235-242.

(53) Wenglowsky, S.; Ahrendt, K. A.; Buckmelter, A. J.; Feng, B.; Gloor, S. L.; Gradl, S.; Grina, J.; Hansen, J. D.; Laird, E. R.; Lunghofer, P.; Mathieu, S.; Moreno, D.; Newhouse, B.; Ren, L.; Risom, T.; Rudolph, J.; Seo, J.; Sturgis, H. L.; Voegtli, W. C.; Wen, Z., Pyrazolopyridine Inhibitors of B-RafV600E. Part 2: Structure-activity Relationships. Bioorg. Med. Chem. Lett. 2011, 21 (18), 5533-7.

(54) Christopher, J. A.; Orgován, Z.; Congreve, M.; Doré, A. S.; Errey, J. C.; Marshall, F. H.; Mason, J. S.; Okrasa, K.; Rucktooa, P.; Serrano-Vega, M. J.; Ferenczy, G. G.; Keserü, G. M., Structure-Based Optimization Strategies for G Protein-Coupled Receptor (GPCR) Allosteric Modulators: a Case Study from Analyses of New Metabotropic Glutamate Receptor 5 (mGlu(5)) X-ray Structures. J. Med. Chem. 2019, 62 (1), 207-222.

(55) Coleman, J. A.; Green, E. M.; Gouaux, E., X-ray Structures and Mechanism of the Human Serotonin Transporter. Nature 2016, 532 (7599), 334-9. 
(56) Cody, V.; Luft, J. R.; Pangborn, W., Understanding the Role of Leu22 Variants in Methotrexate Resistance: Comparison of Wild-type and Leu22Arg Variant Mouse and Human Dihydrofolate Reductase Ternary Crystal Complexes with Methotrexate and NADPH. Acta Crystallogr., Sect. D: Biol. Crystallogr. 2005, 61 (Pt 2), 147-55.

(57) Akwabi-Ameyaw, A.; Bass, J. Y.; Caldwell, R. D.; Caravella, J. A.; Chen, L.; Creech, K. L.; Deaton, D. N.; Jones, S. A.; Kaldor, I.; Liu, Y.; Madauss, K. P.; Marr, H. B.; McFadyen, R. B.; Miller, A. B.; Navas, F., III; Parks, D. J.; Spearing, P. K.; Todd, D.; Williams, S. P.; Wisely, G. B., Conformationally Constrained Farnesoid X receptor (FXR) Agonists: Naphthoic Acid-based Analogs of GW 4064. Bioorg. Med. Chem. Lett. 2008, 18 (15), 4339-43.

(58) Srivastava, A.; Yano, J.; Hirozane, Y.; Kefala, G.; Gruswitz, F.; Snell, G.; Lane, W.; Ivetac, A.; Aertgeerts, K.; Nguyen, J.; Jennings, A.; Okada, K., High-resolution Structure of the Human GPR40 Receptor Bound to Allosteric Agonist TAK-875. Nature 2014, 513 (7516), 124-7.

(59) Thal, D. M.; Sun, B.; Feng, D.; Nawaratne, V.; Leach, K.; Felder, C. C.; Bures, M. G.; Evans, D. A.; Weis, W. I.; Bachhawat, P.; Kobilka, T. S.; Sexton, P. M.; Kobilka, B. K.; Christopoulos, A., Crystal Structures of the M1 and M4 Muscarinic Acetylcholine Receptors. Nature 2016, 531 (7594), 335-40.

(60) Wu, B.; Chien, E. Y. T.; Mol, C. D.; Fenalti, G.; Liu, W.; Katritch, V.; Abagyan, R.; Brooun, A.; Wells, P.; Bi, F. C.; Hamel, D. J.; Kuhn, P.; Handel, T. M.; Cherezov, V.; Stevens, R. C., Structures of the CXCR4 Chemokine GPCR with Small-Molecule and Cyclic Peptide Antagonists. Science 2010, 330 (6007), 1066.

(61) Feldman, J. L.; Dittenhafer-Reed, K. E.; Kudo, N.; Thelen, J. N.; Ito, A.; Yoshida, M.; Denu, J. M., Kinetic and Structural Basis for Acyl-Group Selectivity and NAD(+) Dependence in Sirtuin-Catalyzed Deacylation. Biochemistry 2015, 54 (19), 3037-3050.

(62) Chrencik, J. E.; Roth, C. B.; Terakado, M.; Kurata, H.; Omi, R.; Kihara, Y.; Warshaviak, D.; Nakade, S.; Asmar-Rovira, G.; Mileni, M.; Mizuno, H.; Griffith, M. T.; Rodgers, C.; Han, G. W.; Velasquez, J.; Chun, J.; Stevens, R. C.; Hanson, M. A., Crystal Structure of Antagonist Bound Human Lysophosphatidic Acid Receptor 1. Cell 2015, 161 (7), 1633-43.

(63) Hanson, M. A.; Roth, C. B.; Jo, E.; Griffith, M. T.; Scott, F. L.; Reinhart, G.; Desale, H.; Clemons, B.; Cahalan, S. M.; Schuerer, S. C.; Sanna, M. G.; Han, G. W.; Kuhn, P.; Rosen, H.; Stevens, R. C., Crystal Structure of a Lipid G Protein-coupled Receptor. Science 2012, 335 (6070), 851-5.

(64) Hua, T.; Vemuri, K.; Nikas, S. P.; Laprairie, R. B.; Wu, Y.; Qu, L.; Pu, M.; Korde, A.; Jiang, S.; Ho, J. H.; Han, G. W.; Ding, K.; Li, X.; Liu, H.; Hanson, M. A.; Zhao, S.; Bohn, L. M.; Makriyannis, A.; Stevens, R. C.; Liu, Z. J., Crystal Structures of Agonist-bound Human Cannabinoid Receptor CB(1). Nature 2017, 547 (7664), 468-471.

(65) Cherezov, V.; Rosenbaum, D. M.; Hanson, M. A.; Rasmussen, S. G.; Thian, F. S.; Kobilka, T. S.; Choi, H. J.; Kuhn, P.; Weis, W. I.; Kobilka, B. K.; Stevens, R. C., High-resolution Crystal Structure of an Engineered Human $\beta_{2}$-adrenergic G protein-coupled Receptor Science 2007, 318 (5854), 1258-65.

(66) Stern, E.; Muccioli, G. G.; Millet, R.; Goossens, J. F.; Farce, A.; Chavatte, P.; Poupaert, J. H.; Lambert, D. M.; Depreux, P.; Henichart, J. P., Novel 4-oxo-1,4-dihydroquinoline-3-carboxamide Derivatives as New CB2 Cannabinoid Receptors Agonists: Synthesis, Pharmacological Properties and Molecular Modeling. J. Med. Chem. 2006, 49 (1), 70-9.

(67) Worm, K.; Zhou, Q. J.; Saeui, C. T.; Green, R. C.; Cassel, J. A.; Stabley, G. J.; DeHaven, R. N.; Conway-James, N.; LaBuda, C. J.; Koblish, M.; Little, P. J.; Dolle, R. E., Sulfamoyl Benzamides as Novel CB2 Cannabinoid Receptor Ligands. Bioorg. Med. Chem. Lett. 2008, $18(9), 2830-5$

(68) Pasquini, S.; Mugnaini, C.; Ligresti, A.; Tafi, A.; Brogi, S.; Falciani, C.; Pedani, V.; Pesco, N.; Guida, F.; Luongo, L.; Varani, K.; Borea, P. A.; Maione, S.; Di Marzo, V.; Corelli, F., Design, Synthesis, and Pharmacological Characterization of Indol-3-ylacetamides, Indol3-yloxoacetamides, and Indol-3-ylcarboxamides: Potent and Selective CB2 cannabinoid Receptor Inverse Agonists. J. Med. Chem. 2012, 55 (11), 5391-402.

(69) Tourteau, A.; Andrzejak, V.; Body-Malapel, M.; Lemaire, L.; Lemoine, A.; Mansouri, R.; Djouina, M.; Renault, N.; El Bakali, J.; Desreumaux, P.; Muccioli, G. G.; Lambert, D. M.; Chavatte, P.; Rigo, B.; Leleu-Chavain, N.; Millet, R., 3-Carboxamido-5-aryl-isoxazoles as New CB2 Agonists for the Treatment of Colitis. Bioorg. Med. Chem. 2013, 21 (17), 5383-94.

(70) El Bakali, J.; Muccioli, G. G.; Renault, N.; Pradal, D.; Body-Malapel, M.; Djouina, M.; Hamtiaux, L.; Andrzejak, V.; Desreumaux, P.; Chavatte, P.; Lambert, D. M.; Millet, R., 4-Oxo-1,4-dihydropyridines as Selective CB2 Cannabinoid Receptor Ligands: Structural Insights into the Design of a Novel Inverse Agonist Series. J. Med. Chem. 2010, 53 (22), 7918-7931.

(71) Lu, D.; Meng, Z.; Thakur, G. A.; Fan, P.; Steed, J.; Tartal, C. L.; Hurst, D. P.; Reggio, P. H.; Deschamps, J. R.; Parrish, D. A.; George, C.; Jarbe, T. U.; Lamb, R. J.; Makriyannis, A., Adamantyl Cannabinoids: a Novel Class of Cannabinergic Ligands. J. Med. Chem. 2005, 48 (14), 4576-85.

(72) Li, X.; Hua, T.; Vemuri, K.; Ho, J. H.; Wu, Y.; Wu, L.; Popov, P.; Benchama, O.; Zvonok, N.; Locke, K.; Qu, L.; Han, G. W.; Iyer, M. R.; Cinar, R.; Coffey, N. J.; Wang, J.; Wu, M.; Katritch, V.; Zhao, S.; Kunos, G.; Bohn, L. M.; Makriyannis, A.; Stevens, R. C.; Liu, Z. J., Crystal Structure of the Human Cannabinoid Receptor CB2. Cell 2019, 176 (3), 459-467.

(73) Rinaldi-Carmona, M.; Barth, F.; Millan, J.; Derocq, J.-M.; Casellas, P.; Congy, C.; Oustric, D.; Sarran, M.; Bouaboula, M.; Calandra, B.; Portier, M.; Shire, D.; Brelière, J.-C.; Fur, G. L., SR 144528, the First Potent and Selective Antagonist of the CB2 Cannabinoid Receptor J. Pharmacol. Exp. Ther. 1998, 284 (2), 644-650. 